\title{
Nématodes Trichostrongyloidea Parasites de Microchiroptères
}

\author{
par M.-C. DURETTE-DESSET et A.-G. CHABAUD \\ Laboratoire de Zoologie (Vers) associé au C.N.R.S. \\ Muséum national d'Histoire naturelle, 43, rue Cuvier, F 75231 Paris Cedex 05.
}

\section{Résumé.}

1. a) Liste des Nématodes, parasites de Chiroptères européens récoltés par le $\mathrm{P}^{\mathrm{r}}$ Aellen : compléments à l'étude morphologique de Molinostrongylus alatus, M. panousei, M. skrjabini ; description de $M$. aelleni $\mathrm{n}$. sp.

b) Description de $M$. richardae n. sp., $M$. benexae n. sp. et $M$. bauchoti n. sp. parasites de Molossidae malgaches.

c) Description de $M$. colleyi n.sp. et $M$. owyangi n. sp., parasites de Vespertilioninae malais, et d'Allintoschius dunni n. sp., trouvé chez Myotis mystacinus en Malaisie et Pipistrellus nanus en Afrique.

2. D'après le syn!ophe, les 17 espèces du genre Molinostrongylus semblent se répartir en cinq groupes d'espèces, chacun d'eux étant assez caractéristique d'un genre déterminé de Chiroptères.

3. La composition de la nématofaune (Trichostrongyloidea) des Chiroptères est analysée, ainsi que ses rapports avec les Trichostrongyloidea d'autres Mammifères (Tupaiidae, Pholidotes, Primates, Sciuridés). Six groupes sont individualisés, constituant deux ensembles bien distincts: genre Strongylacantha, d'une part; 12 genres plus ou moins directement issus des Molineinae, d'autre part.

4. Les trois pointes de l'extrémité caudale des femelles, qui définissent actuellement les Anoplostrongylinae par rapport aux Molineinae, se révèlent un caractère insuffisant. Les deux sous-familles forment un ensemble complexe, qui ne pourra être interprété qu'en tenant compte également de l'évolution du synlophe et de celle des bourses caudales des mâles. 


\section{Summary.}

Trichostrongyloidea Nematodes parasites from Microchiroptera.

1. a) List of Nematodes collected by Professor Aellen in european Microchiroptera. Additionnal morphological data to the study of Molinostrongylus alatus, M. panousei, $M$. skrjabini. Description of $M$. aelleni n. sp.

b) Description of $M$. richardae n. sp., $M$. benexae n. sp. et $M$. bauchoti n. sp., parasites of malagasian Molossidae.

c) Description of $M$. colleyi n. sp. and $M$. owyangi n. sp., parasites of Malaysian Vespertilioninae, and of Allintoschius dunni n. sp., discovered in Myotis mystacinus from Malaysia and Pipistrellus nanus from Africa.

2. Taking into account the characieristics of the synlophe, the 17 species of the genus Molinostrongylus may be divided into five groups, each one being reasonably well characteristic of the genus of their Chiropteran host.

3. The composition of the Trichostrongyloidea fauna of Chiroptera and its relationship with Trichostrongyloidea from other Mammals (Tupaiidae, Pholidotes, Primates, Sciuridés) are analysed. Six groups are separated and divided into two well defined lines: 1) genus Strongylacantha, and 2) 12 genera stemming more or less directly from the Molineinae.

4. The three conical outgrowths at the tip of the female tail which differenciate presently the Anoplostrogylinae from the Molineinae appear to be an unreliable characteristic. The two subfamilies form a complex group which will be better understood if the evolution of the synlophe and that of the caudal bursa of the males are taken into account.

\section{I. - Etude des parasites de Chiroptères européens (Collection Aellen)}

\section{A) Répartition des espèces selon les hôtes.}

- Miniopterus natalensis Smith. $1192 \mathrm{Ka}$, grotte de Baya, Katanga, 12-3-1956: nombreux ơ, \& Litomosa chiropterorum Ortlepp, 1932 (1).

- Myotis myotis Bechstein. 1178 Ka, grotte de Pertuis, Neuchâtel, Suisse, 16-2-1962 : 2 ㄴ Litomosa desportesi Bain, 1967.

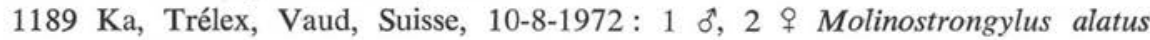
(Ortlepp, 1932).

- Myotis blythii oxygnathus (Monticelli). $1190 \mathrm{Ka}$, Zagorska pec, Novi, Yougoslavie, 9-9-1972: 1 ơ, 5 \& Molinostrongylus alatus (Ortlepp, 1932).

$1191 \mathrm{Ka}, I d$. : + éclatées qui semblent identifiables à $M$. alatus (Ortlepp, 1932).

2 ㅇ Capillaria sp.

- Myotis bechsteini (Leisler). $1167 \mathrm{Ka}$, Commugny, Vaud, Suisse, 6-8-1958 : 1 ð, 5 \& Molinostrongylus alatus (Ortlepp, 1932).

(1) Cité ici, le tube faisant partie de la collection. 
- Myotis nattereri (Kuhl). $1174 \mathrm{Ka}$, Commugny, Vaud, Suisse, 9-9-1961: 1 ð, 1 우 Molinostrongylus alatus (Ortlepp, 1932).

- Myotis mystacinus (Kuhl). $1170 \mathrm{Ka}$, grotte inférieure de Vallorbe, Vaud, Suisse, 25-12-1958 : 5 \& Rictularia bovieri (Blanchard, 1886).

$1176 \mathrm{Ka}$, grotte supérieure de Vallorbe, Vaud, Suisse, 24-1-1962: 8 \& Rictularia bovieri (Blanchard, 1886).

$1183 \mathrm{Ka}$, Col de Jaman, Vaud, Suisse, 17-9-1967: 4 q Rictularia bovieri (Blanchard, 1886).

- Plecotus auritus L. 1172 Ka, Commugny, Vaud, Suisse, 9-9-1961 : 1 \& Seuratum mucronatum (Rud., 1809).

$1173 \mathrm{Ka}$, Id. : 1 o , 3 \& Seuratum mucronatum (Rud., 1809).

$1175 \mathrm{Ka}$, grotte Chaudière d'Enfer, Vaud, Suisse, 24-12-1961: 2 , 1 đ juv. Litomosa filaria (Rud., 1809).

$1184 \mathrm{Ka}$, Col de Jaman, Vaud, Suisse, 17-9-1967: 1 Litomosa filaria (Rud., 1809).

- Plecotus austriacus Fisher. 1188 Ka, Genève, Suisse, 20-5-1971: 1 \& Litomosa filaria (Beneden, 1873).

- Pipistrellus nathusii (Keyserling et Blasius). $1181 \mathrm{Ka}$, Col de Jaman, Vaud, Suisse, 17-9-1967: 2 q Molinostrongylus sp. Le synlophe est identique à celui de $M$. skjrabini.

- Nyctalus leisleri (Kuhl). $1182 \mathrm{Ka}, \mathrm{Col}$ de Jaman, Vaud, Suisse, 17-9-1967: 3 q Molinostrongylus sp. Synlophe type M. skrjabini, mais les ailes latérales disparaissent au niveau du tiers antérieur et non dans la partie postérieure du corps. Il s'agit vraisemblablement d'une espèce nouvelle.

- Nyctalys noctula (Schreber). $1168 \mathrm{Ka}$, Uster, Zurich, Suisse, 3-12-1958 : nombreux $\sigma^{t}$ et $q$ Molinostrongylus skrjabini Skarbilovitsch, 1934.

$1169 \mathrm{Ka}, \mathrm{Id}$. : nombreux ơ et + Molinostrongylus skrjabini Skarbilovitsch, 1934 $1171 \mathrm{Ka}, \mathrm{Col}$ de Bretolet, Valais, 26-8-1959: 2 \& Molinostrongylus skrjabini Skarbilovitsch, 1934.

$1185 \mathrm{Ka}, \mathrm{Col}$ de Jaman, Vaud, Suisse, 17-9-1967: 2 ơ, 5 Molinostrongylus skrjabini Skarbilovitsch, 1934.

- Nyctalus lasiopterus (Schreber). $1179 \mathrm{Ka}$, Col de Bretolet, Valais, Suisse, 2-101962 : nombreux ot et +9 Molinostrongylus aelleni $\mathrm{n}$. sp.

$1180 \mathrm{Ka}$, Id., 1-10-1962 : nombreux ô et $\$$ Molinostrongylus aelleni $\mathrm{n}$. sp. types.

- Vespertilio murinus Schreber. 1177 Ka, Bellerive, Genève, Suisse, 3-5-1962 :

1 Litomosa sp., $\mathrm{n}^{\circ} 1$ (1).

$1187 \mathrm{Ka}$, Col de Jaman, Vaud, Suisse, 17-9-1967: 1 \& Litomosa sp., $\mathrm{n}^{\circ} 1$.

- Tadarina teniotis Rafinesque. $1166 \mathrm{Ka}$, Col de Bretolet, Valais, Suisse, 11-8-1958 :

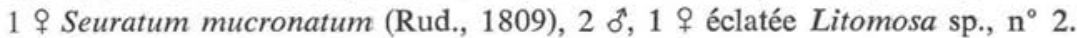

(1) Litomosa sp. $\mathrm{n}^{\circ} 1$ et Litomosa sp. $\mathrm{n}^{\circ} 2$ correspondent toutes deux à des espèces non encore décrites. Le matériel disponible ne permettra cependant que la description de Litomosa sp. $\mathrm{n}^{\circ} 2$. 


\section{B) Etude des Trichostrongyloidea parasites de Chiroptères européens.}

\section{Molinostrongylus alatus (Ortlepp, 1932)}

MAtÉRIel utilisé : $1 \delta^{*}, 2$ parasites de Myotis myotis, Bechstein $\mathrm{n}^{\circ} 3421$, originaire de Suisse, Trélex, Vaud.

Synlophe: chez les deux sexes, corps parcouru longitudinalement par 8 arêtes cuticulaires, 3 ventrales et 3 dorsales petites, plus 2 ailes latérales hautes d'environ $20 \mu$ (fig. $1, C$ ). Les crêtes naissent sur le bord postérieur de la vésicule céphalique Dans la partie postérieure du corps, environ $300 \mu$ en avant de la bourse caudale chez le $\delta$, entre la vulve et l'anus chez la $q$, une arête supplémentaire apparaît sur chaque face.

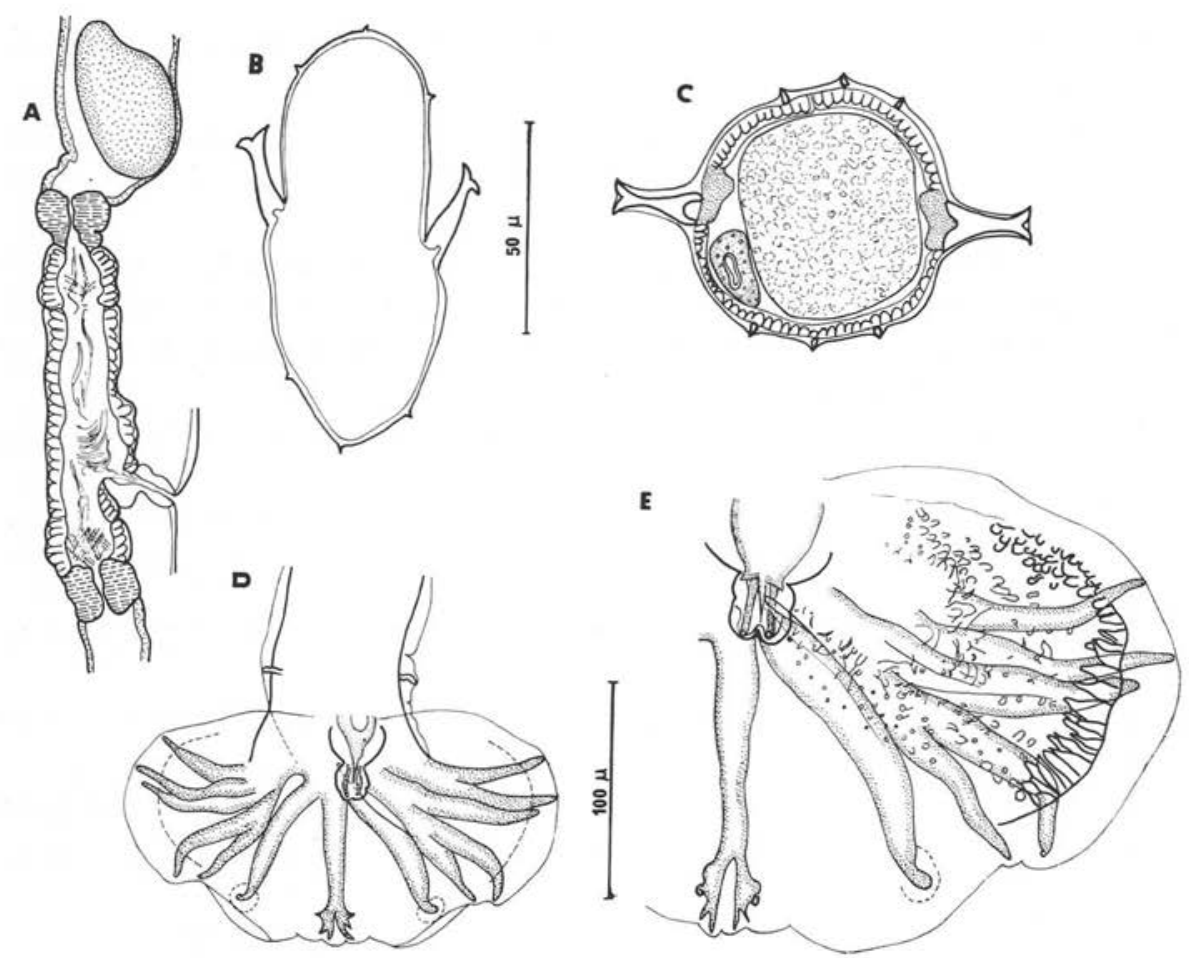

FIG. 1. - Molinostrongylus alatus (Ortlepp, 1932). A : $\$$, ovéjecteur, vue latérale droite; B :

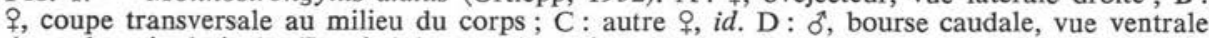
(sans la spinulation); E : $\delta$, lobe gauche, détail de la spinulation, vue ventrale. (B, d'après le matériel marocain).

A, D : éch. $100 \mu$; B, C, E : éch. $50 \mu$. 
Principales mensurations.

Mâle: longueur : $2,8 \mathrm{~mm}$; largeur : $65 \mu$; vésicule céphalique : $35 \mu \times 30 \mu$; anneau nerveux, pore excréteur, déirides situés respectivement à $160 \mu, 182 \mu$ et $192 \mu$. de l'apex. Esophage : $280 \mu$; spicules : $275 \mu$; gubernaculum : $70 \mu$. Bourse caudale figurée en $1, D, E$.

Femelle: longueur : $3 \mathrm{~mm}$; largeur : $100 \mu$ (ailes comprises); vésicule céphalique : $35 \mu \times 30 \mu$; anneau nerveux, pore excréteur, déirides situés respectivement à $160 \mu, 180 \mu$ et $190 \mu$ de l'apex. Esophage : $260 \mu$. Queue : $60 \mu$; vulve située à $725 \mu$ de la pointe caudale ; vagina vera: $28 \mu$; branche antérieure de l'ovéjecteur : vestibule $78 \mu$, sphincter $29 \mu$, trompe $25 \mu$; branche postérieure de l'ovéjecteur: vestibule $10 \mu$, sphincter $22 \mu$, trompe $21 \mu$. Eufs: $72 \mu \times 40 \mu$ (fig. 1, A).

vestibule $10 \mu$,

\section{Discussion.}

La dorsale est parfois longue, parfois très longue, et la longueur des spicules varie également dans de très larges proportions. Entre le matériel du Maroc étudié par Dollfus, 1954 (dorsale très longue, spicules de $340 \mu$ ), et le matériel de Suisse (dorsale plus courte, spicules de $225 \mu$ à $280 \mu$.), nous n'avons trouvé de différences ni dans le synlophe (fig. 1, B, C), ni dans la pointe des spicules. Nous pensons donc qu'il s'agit d'une seule espèce polymorphe.

\section{Molinostrongylus panousei Dollfus, 1954}

La redescription est faite d'après des documents non publiés de Camille Desportes sur du matériel de Miniopterus schrebersi Kuhl, grotte de Pouade (Pyrénées-Orientales), France.

Synlophe: chez les deux sexes, corps parcouru longitudinalement par 16 arêtes cuticulaires, dont deux ailes latérales larges de $20 \mu$. environ, 7 arêtes ventrales, 7 arêtes dorsales (fig. 2, A). Les ailes naissent au voisinage des déirides et s'étendent jusqu'au niveau de la bourse caudale chez le $\sigma^{t}$ et de la vulve chez la $q$. Chez cette dernière, au-delà de la vulve, on trouve 9 arêtes dorsales et 11 ventrales qui s'anastomosent au niveau de la queue.

\section{Principales mensurations.}

Mâle: longueur : 3,5 mm ; largeur: $72 \mu$ (ailes comprises). Vésicule céphalique : $50 \mu \times 20 \mu$. Anneau nerveux, pore excréteur et déirides situés respectivement à $165 \mu$, $225 \mu$. et $225 \mu$. de l'apex. Esophage : $400 \mu$. Spicules : $225 \mu$ (fig. 2, D). Gubernaculum : $80 \mu$. Bourse caudale figurée en $2, B, C$.

Femelle: longueur: $4,5 \mathrm{~mm}$; largeur : $120 \mu$ (ailes comprises). Queue : $42 \mu$ (fig. 2, G). Vulve située à $1,7 \mathrm{~mm}$ de la pointe caudale. Branche postérieure de l'ovéjecteur atrophiée. Longueur totale entre les 2 sphincters : $200 \mu$. Eufs : $90 \mu \times 44 \mu$. 


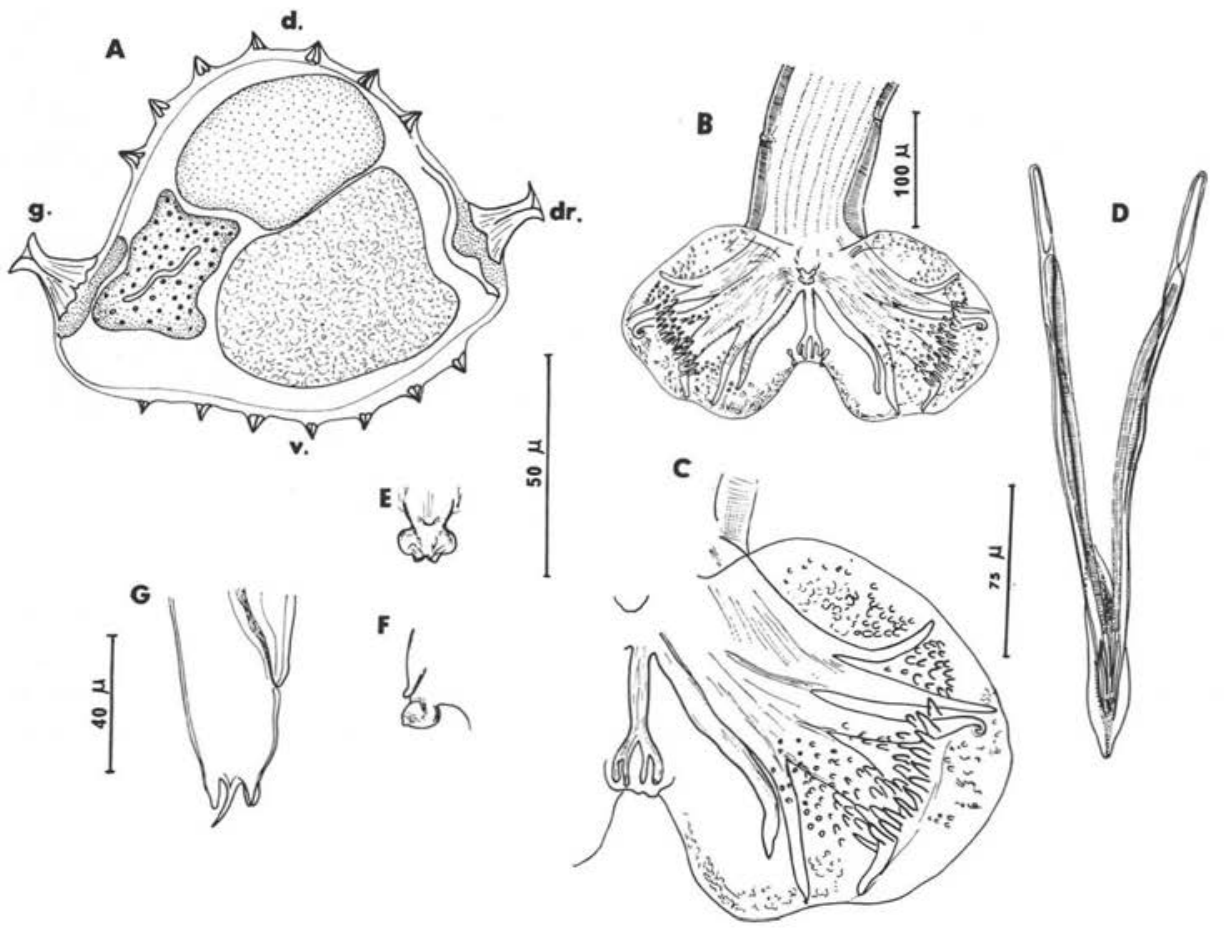

Fig. 2. - Molinostrongylus panousei Dollfus, 1954. A: coupe transversale au milieu du corps; $\mathrm{B}: \delta^{2}$, bourse caudale, vue ventrale; $\mathrm{C}: \delta^{\alpha}$, lobe gauche de la bourse caudale, détail de la spinulation, vue ventrale; D : $\delta$, spicules et gubernaculum; E, F : ô, cône génital, successivement vues ventrale et latérale gauche; G: + , extrémité postérieure, vue latérale droite.

A : éch. $50 \mu$; B : éch. $100 \mu$; C, D, E, F : éch. $75 \mu$; G : éch. $40 \mu$. (B-G: d'après Desportes, dessins inédits Faune de France).

\section{Discussion.}

L'espèce a été étudiée par C. Desportes, qui l'avait alors déterminée comme M. ornatus (Mönnig, 1927). Nous supposons qu'il s'agit d'une espèce différente, bien que très proche, car Ortlepp, en 1932, a décrit le synlophe de la forme sud-africaine comme ayant deux ailes latérales, deux ailes médianes et trois crêtes intermédiaires sur chacune des faces latéro-médianes. Sur le matériel européen, au contraire, les deux ailes latérales sont très développées, mais les lignes ventrale et dorsale portent une arête qui ne se distingue pas de celles des faces latéro-médianes (fig. 2, A).

Nous avons pu vérifier que le matériel de France est identique à celui du Maroc décrit par Dollfus en 1945, sous le nom de $M$. panousei. Ce dernier, travaillant sur préparation montée, a confondu la côte dorsale et les papilles de la lèvre postérieure du cône génital. Il existe, en réalité, une côte dorsale aussi bien développée que sur 
le matériel de Desportes, et M. Dollfus nous a demandé de faire, en son nom, la rectification nécessaire.

\section{Molinostrongylus skrjabini Skarbilovitsch, 1934}

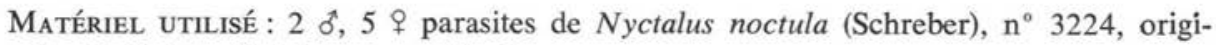
naire de Suisse, Col de Jaman, Vaud.

Synlophe : chez les deux sexes, corps parcouru longitudinalement par deux ailes cuticulaires naissant derrière la vésicule céphalique et disparaissant juste en arrière du niveau de la vulve chez la $q$ et à environ $180 \mu$ en avant de la bourse caudale chez le ${ }^{\top}$. Les faces ventrale et dorsale sont dépourvues d'arêtes (fig. 3, A).

\section{Principales mensurations.}

Mâle : longueur : 2,4 mm ; largeur : $57 \mu$ (ailes comprises). Vésicule céphalique : $22 \mu \times 20 \mu$. Anneau nerveux, pore excréteur et déirides situés respectivement à $95 \mu$, $115 \mu$. et $100 \mu$ de l'apex. Esophage : $170 \mu$. Spicules : $120 \mu$ (fig. 3, F). Bourse caudale figurée en $3, E$.

Femelle : longueur : $3 \mathrm{~mm}$; largeur : $65 \mu$ (ailes comprises). Vésicule céphalique : $22 \mu \times 20 \mu$. Anneau nerveux, pore excréteur et déirides situés respectivement à $78 \mu$, $90 \mu$ et $95 \mu$ de l'apex. Esophage : $170 \mu$. Queue : $50 \mu$ (fig. 3, B, C). Vulve située à $460 \mu$ de la pointe caudale. Vagina vera: $26 \mu$. Branche antérieure de l'ovéjecteur : vestibule, $45 \mu$; sphincter, $19 \mu$; trompe, $24 \mu$. Branche postérieure de l'ovéjecteur : vestibule, $19 \mu$; sphincter, $11 \mu$; trompe, $14 \mu$. Eufs : $80 \mu \times 40 \mu$ (fig. 3, D).

\section{Discussion.}

L'espèce possède deux éléments particuliers : d'une part, les spicules se terminent par une pointe simple; d'autre part, le synlophe est constitué uniquement de deux ailes latérales. En plus de $M$. aelleni n. sp. décrit ci-après, il existe certainement d'autres espèces proches. Ainsi, chez un Nyctalus leisleri, nous avons trouvé trois femelles de Molinostrongylus sp. de synlophe comparable, mais les arêtes latérales s'arrêtent au tiers antérieur du corps, alors que, chez $M$. skrjabini, elles se poursuivent jusqu'au niveau de la vulve. Il s'agit donc certainement d'une espèce différente, dont nous ne connaissons pas le mâle. Chez un Pipistrellus nathusii (Keyserling et Blasius), nous avons trouvé également deux femelles de Molinostrongylus sp. avec le même type de synlophe (fig. 11, E), mais cette fois les ailes latérales s'arrêtent au niveau de la vulve et, en l'absence de mâle, nous ne pouvons savoir si elles diffèrent ou non de $M$. skriabini. 

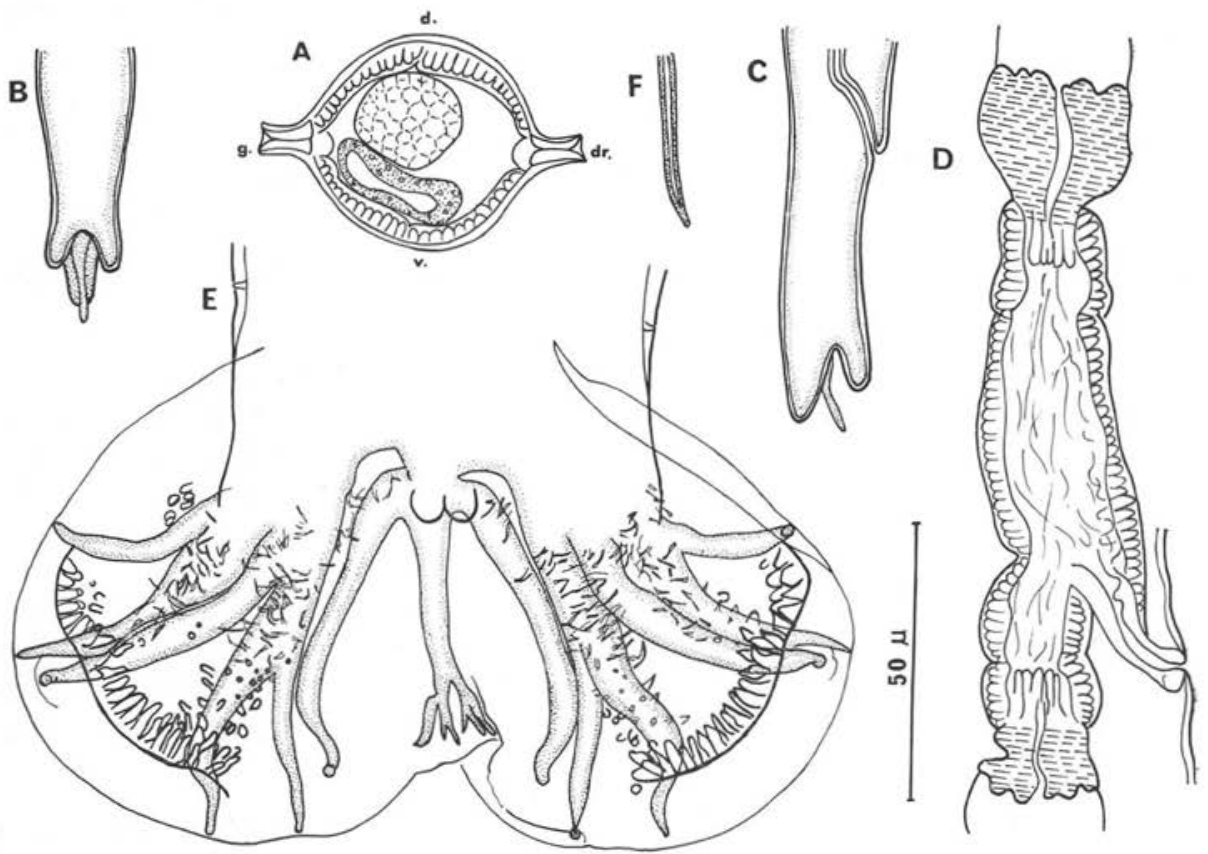

FIG. 3. - Molinostrongylus skrjabini Skarbilovitsch, 1934. A: + , coupe transversale au milieu du corps; B : + , extrémité caudale, vue ventrale; C: + , extrémité postérieure, vue latérale droite; D: $q$, ovéjecteur, vue latérale droite; E: $\delta$, bourse caudale, vue ventrale ; F : $\delta$, pointe d'un spicule.

\section{Molinostrongylus aelleni $\mathrm{n} . \mathrm{sp}$.}

MATÉRIEL TYPE : nombreux ơ et $q, \mathrm{n}^{\circ} 1180 \mathrm{Ka}$.

Hôte: Nyctalus lasiopterus (Schreber), $\mathrm{n}^{\circ} 2684$.

LOCALISATION : intestin grêle.

ORIGINE GÉOGRAPHIQUE: Col de Bretolet, Valais, Suisse.

\section{DESCRIPTION.}

Le corps est déroulé. En vue apicale, la tête porte 2 amphides, 4 papilles labiales externes et 4 papilles céphaliques. La bouche est grossièrement triangulaire (fig. $4, B$ ) ; les glandes excrétrices sont très visibles (fig. 4, A). Pore excréteur et déirides situés au même niveau, en arrière de l'anneau nerveux. Canal excréteur court.

Synlophe: chez les deux sexes, corps parcouru longitudinalement par deux arêtes latérales hautes d'environ $15 \mu$, qui débutent sur le bord postérieur de la vésicule céphalique et disparaissent un peu en arrière de la vulve chez la $q$ (fig. $4, E$ ) et aux $9 / 10$ de la longueur totale chez le ơ. 


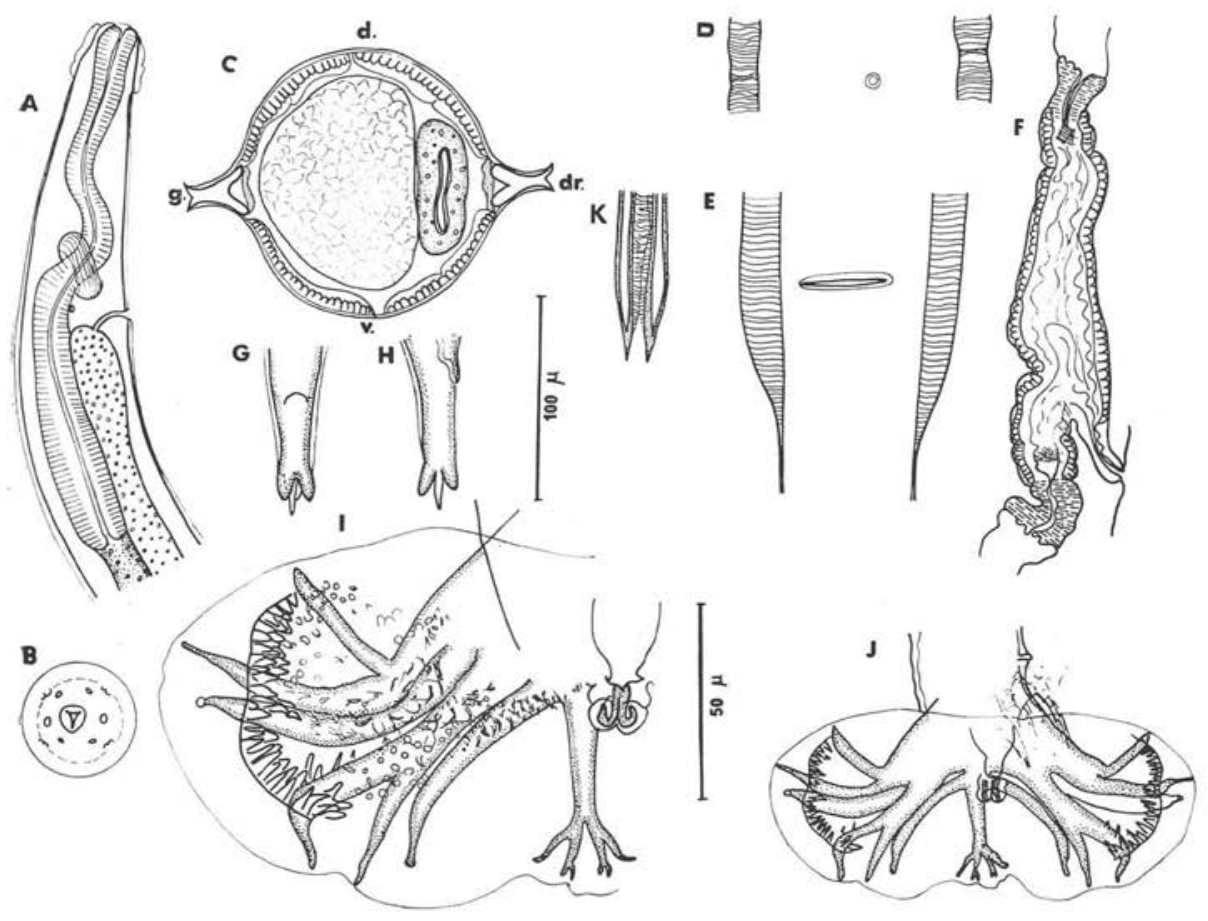

FIG. 4. - Molinostrongylus aelleni n. sp. A: $\$$, extrémité antérieure, vue latérale droite;

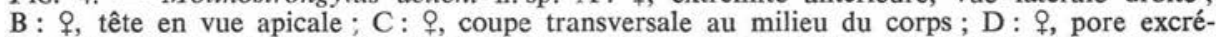
teur et deirides, vue ventra!e; E: + , région vulvaire, fin des arêtes cuticulaires, vue ventrale ; F : $\{$, ovéjecteur, vue latérale droite; G, H: $q$, extrémité postérieure, vues ventrale et latérale droite; $\mathrm{I}: \delta$, lobe droit de la bourse caudale avec détail de la spinulation, vue ventrale ; $\mathbf{J}: \delta^{*}$, bourse caudale, vue ventrale; $\mathrm{K}: \delta^{\prime}$, pointe des spicules.

$\mathrm{A}, \mathrm{E}, \mathrm{F}, \mathrm{G}, \mathrm{H}, \mathrm{J}$ : éch. $100 \mu$; B, C, D, I, K: éch. $50 \mu$.

Mâle: corps long de $3 \mathrm{~mm}$, large de $50 \mu$ dans sa partie moyenne (80 $\mu$ arêtes comprises). Vésicule céphalique haute de $26 \mu$ sur $29 \mu$ de large. Anneau nerveux, pore excréteur et déirides situés respectivement à $110 \mu, 110 \mu$ et $100 \mu$ de l'apex. Esophage long de $232 \mu$. Bourse caudale figurée en 4, I, J. Papilles prébursales situées à $30 \mu$ en avant de la bourse caudale. Spicules à pointe simple (fig. $4, K$ ), longs de $410 \mu$ (390 $\mu$ chez un autre spécimen). Cône génital haut de $23 \mu$. Papille 0 pointue, papilles 7 foliacées (fig. 4, I). Gubernaculum haut de $80 \mu$.

Femelle : corps long de $4,3 \mathrm{~mm}$, large de $65 \mu$ dans sa partie moyenne $(95 \mu$ arêtes comprises). Vésicule céphalique haute de $32 \mu$ sur $32 \mu$ de large. Anneau nerveux, pore excréteur et déirides situés respectivement à $115 \mu, 142 \mu$ et $140 \mu$ de l'apex (déiride gauche), $134 \mu$ (déiride droite). Esophage long de $270 \mu$ (fig. 4, A). Queue : $60 \mu$, avec un filament caudal long de $19 \mu$. Trois pétales caudaux, le dorsal étant sensiblement de même longueur que les deux latéro-ventraux (fig. $4, G, H$ ). Vulve 
s'ouvrant à $800 \mu$ de la pointe caudale. Vagina vera long de $32 \mu$. Branche antérieure de l'ovéjecteur : vestibule, $125 \mu$; sphincter, $22 \mu$; trompe, $22 \mu$. La branche postérieure de l'ovéjecteur est très réduite, comme chez la plupart des Molinostrongylus, cette réduction portant essentiellement sur la longueur du vestibule. Vestibule, $18 \mu$; sphincter, $20 \mu$; trompe, $38 \mu$. Eufs hauts de $80 \mu$ sur $32 \mu$ de large (fig. $4, F$ ).

\section{Discussion.}

Par son synlophe et ses spicules à pointe simple, l'espèce est à rapprocher du groupe skrjabini. Dans ce groupe, la seule espèce dont les spicules soient relativement longs $(280 \mu)$ est $M$. longispiculus (Yamashita et Mori, 1953) Andreiko et coll., 1968, parasite de Nyctalus maximus au Japon. Nos spécimens ont des spicules longs de $390 \mu$. à $410 \mu$. Par ailleurs, certaines différences avec l'espèce japonaise apparaissent sur les dessins de la bourse caudale. La côte 4 , en particulier, est plus longue que la 3 chez longispiculus, plus courte chez nos spécimens. Nous considérons donc qu'il s'agit d'une espèce nouvelle, que nous nommons Molinostrongylus aelleni n. sp. en la dédiant à M. le $\mathrm{P}^{\mathrm{r}}$ V. Aellen.

\section{II. - Etude des Trichostrongyloidea parasites de Chiroptères malgaches}

\section{Molinostrongylus richardae n. sp.}

MATÉRIEl TYPE : 3 o*, 9 \&, $\mathrm{n}^{\circ} 291 \mathrm{H}$.

HôTE: Mormopterus albiventer (Dobson).

LOCALISATION : intestin.

ORIGINE GÉOGRAPHIQUe: Anjiro, 30-11-1966, Madagascar.

L'espèce a été retrouvée chez quatre Mormopterus albiventer de la même région ( $276 \mathrm{H}, 277 \mathrm{H}, 282 \mathrm{H}, 301 \mathrm{H}$ ) et chez un Eptesicus pusillus (Le Conte) originaire de Tananarive.

\section{DESCRIPTION.}

Le corps est déroulé. En vue apicale, la tête porte 2 amphides, 4 papilles labiales externes et 4 papilles céphaliques. La bouche est de forme triangulaire (fig. $5, B$ ). Vésicule céphalique allongée, pore excréteur situé antérieurement à l'anneau nerveux ; 
canal excréteur très long, glandes excrétrices bien visibles, déirides très postérieures (fig. $5, A$ ). Chez le mâle, les papilles prébursales sont très marquées (fig. $5, J$ ) ; le cône génital très complexe (fig. $5, M$ ). Chez la femelle, contrairement à la plupart des autres Molinostrongylus, la branche postérieure de l'ovéjecteur n'est pas atrophiée (fig. $5, G)$. Queue avec 3 pétales caudaux, le dorsal étant plus grand que les 2 latéroventraux (fig. 5, $\mathrm{H}$ ).

Synlophe: chez les deux sexes, corps parcouru longitudinalement par 2 comarêtes latérales (ailes), hautes d'environ $20 \mu, 3$ comarêtes ventrales et 3 comarêtes dorsales. Les ailes débutent au niveau du pore excréteur, les autres arêtes à peu près au niveau de l'anneau nerveux et s'étendent jusqu'au niveau de la bourse caudale chez le $\delta$ (fig. $5, J$ ). Chez la $\uparrow$, les ailes diminuent brusquement de hauteur juste en arrière de la vulve (fig. $5, F$ ) et disparaissent, comme les autres arêtes, entre le niveau de la vulve et l'extrémité caudale. Un jeune adulte femelle, encore dans la cuticule du quatrième stade larvaire, nous a permis d'observer que ce stade était dépourvu d'arêtes cuticulaires (fig. 5, C).

Mâle : corps long de 4,5 mm, large de $150 \mu$ dans sa partie moyenne, ailes comprises. Vésicule céphalique haute de $70 \mu$ sur $35 \mu$ de large. Anneau nerveux, pore excréteur et déirides situés respectivement à $160 \mu$, $115 \mu$ et $280 \mu$ de l'apex. Canal excréteur : $70 \mu$. Esophage long de $335 \mu$ (fig. 5, A). Bourse caudale figurée en $5, \mathrm{~J}$. Papilles prébursales à $120 \mu$ en avant de la bourse caudale. Spicules ailés, longs de $200 \mu$, à extrémité distale bifide (fig. $5, K, L$ ). Les pointes des deux spicules n'ont pu être séparées après dissection. Gubernaculum haut de $86 \mu$ sur $24 \mu$ de large (fig. $5, N$ ). Cône génital haut de $45 \mu$ sur $30 \mu$ de large. Papille 0 en forme de languette. Papilles 7 allongées (fig. 5, M).

Femelle: corps long de 7,9 mm, large de $160 \mu$ dans sa partie moyenne (ailes comprises). Vésicule céphalique haute de $75 \mu$ sur $40 \mu$ de large. Anneau nerveux, pore excréteur et déirides situés respectivement à $180 \mu, 120 \mu$ et $240 \mu$ de l'apex. Canal excréteur long de $90 \mu$. Esophage long de $450 \mu$. Queue : $82 \mu$; filament caudal : $20 \mu$ (fig. $5, H, I$ ). Vulve s'ouvrant à $1,75 \mathrm{~mm}$ de la pointe caudale. Didelphie. Vagina vera: $23 \mu$. Branche antérieure de l'ovéjecteur: vestibule, $65 \mu$; sphincter, $28 \mu$; trompe, $26 \mu$; branche postérieure de l'ovéjecteur : vestibule, $59 \mu$; sphincter, $22 \mu$; trompe, $21 \mu$ (fig. 5, G). La branche utérine antérieure mesure 1,6 mm, postérieure $1,3 \mathrm{~mm}$. Eufs hauts de $70 \mu$ sur $40 \mu$ de large.

\section{Discussion.}

Pour les raisons que nous exposerons plus loin (voir discussion sur Molinostrongylus bauchoti), nous considérons que cette espèce est nouvelle et nous la nommons Molinostrongylus richardae $\mathrm{n}$. sp., en la dédiant à notre collègue, $\mathbf{M}^{\mathrm{me}}$ Josette Richard, qui a récolté l'espèce. 


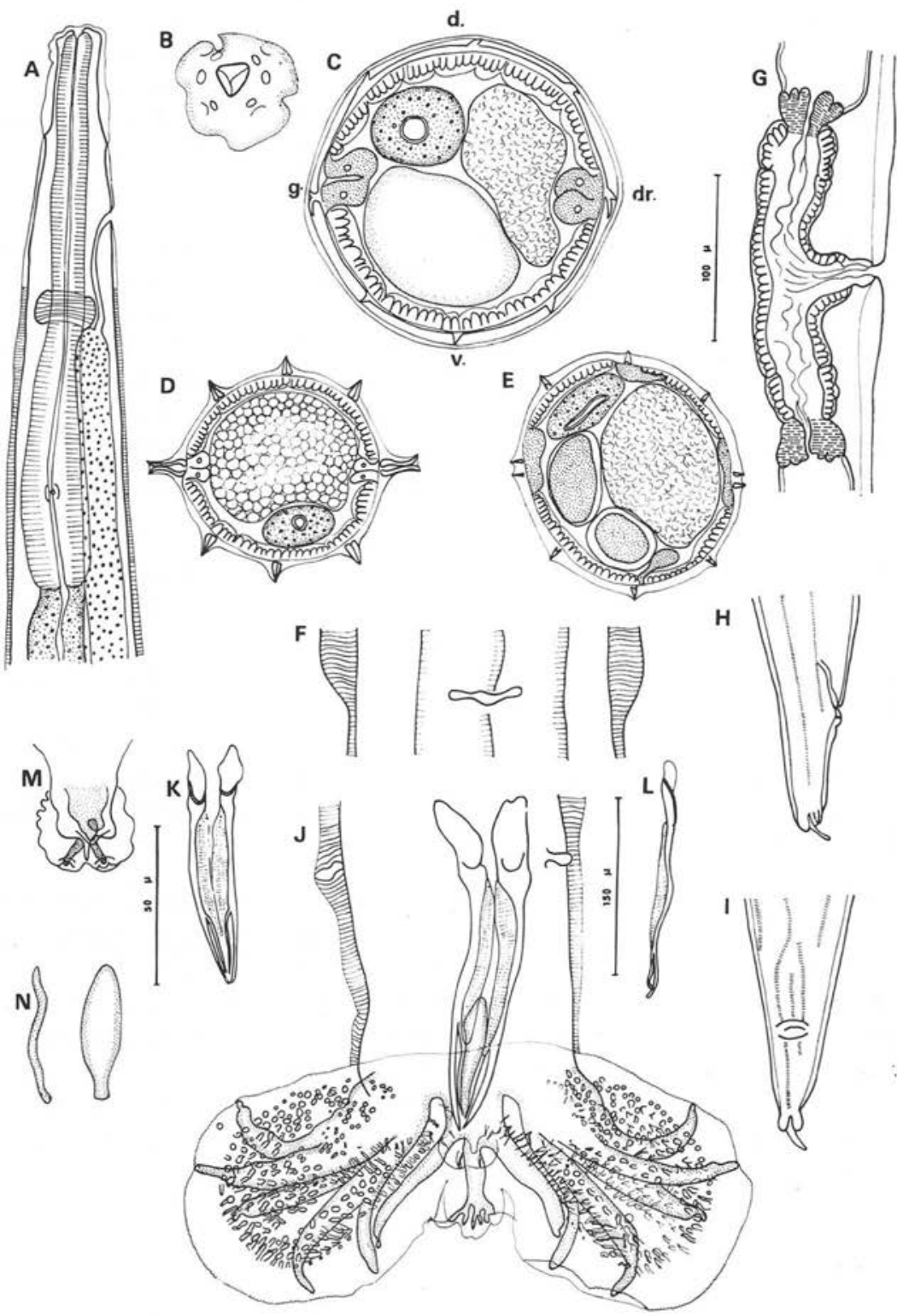

FIG. 5. - Molinostrongylus richardae n. sp. A: $\delta^{*}$, extrémité antérieure, vue latérale droite; B : $q$, tête en vue apicale; $\mathrm{C}$ : jeune adulte $\$$, dans la mue du $4^{\circ}$ stade larvaire, coupe transversale au tiers antérieur du corps; D: $q$, coupe transversale au milieu du corps; E : $i d$., entre vulve et anus; F : 9 , région vulvaire, détail des arêtes cuticulaires, vue ventrale ; G: + , ovéjecteur, vue latérale droite ; $\mathrm{H}: \mathcal{q}$, extrémité postérieure, vue latérale droite ; I : $i d$., vue ventrale; $\mathbf{J}: \delta^{*}$, bourse caudale, vue ventrale; $\mathrm{K}, \mathrm{L}: \delta$, spicules vus de face et de profil; $\mathrm{M}: \delta^{*}$, cône génital, vue ventrale; $\mathrm{N}: \delta^{*}$, gubernaculum, vues latérale droite et dorsale.

A, D, E, F, G, H, I, J : éch. $100 \mu ; \mathrm{B}, \mathrm{C}, \mathrm{M}, \mathrm{N}$ : éch. $50 \mu ; \mathrm{K}, \mathrm{L}$ : éch. $150 \mu$. 
Molinostrongylus benexae $\mathrm{n} . \mathrm{sp}$.

MATÉRIEL TYPE : 9 ơ, 4 \%, n $\mathrm{n}^{\circ} 692$ E.

Hôte : Chaerophon limbatus (Peters).

LOCALISATION : intestin.

OrIGINE GÉOGRAPHIQUE : Périnet, Madagascar. (Grenier de la maison de passage de la station forestière).

\section{DESCRIPTION.}

Le corps est déroulé. En vue apicale, la tête est identique à celle de $M$. richardae. Vésicule céphalique allongée, pore excréteur situé antérieurement à l'anneau nerveux ; canal excréteur très long, glandes excrétrices bien visibles, déirides très postérieures (fig. 6, A). Chez la + , queue avec trois pétales caudaux, le dorsal étant plus grand que les deux latéro-ventraux (fig. 6, I).

Synlophe: corps parcouru longitudinalement par 6 comarêtes (3 ventrales, 3 dorsales) chez la $\$$ (fig. $6, C$ ), 10 (5 ventrales, 5 dorsales) chez le $\delta^{t}(f i g .6, B$ ) et 2 comarêtes latérales (ailes). Les ailes, hautes de $11 \mu$ chez le $\delta^{\top}$ et $18 \mu$ chez la $q$, débutent au niveau du pore excréteur et les autres comarêtes au niveau de l'anneau nerveux. Les premières disparaissent au niveau de la vulve chez la $\varsubsetneqq$ (fig. $6, F$ ); à ce niveau, il n'existe plus qu'une seule arête ventrale et trois arêtes dorsales (fig. $6, D$ ). Entre la vulve et l'anus, le nombre des arêtes atteint 14 , mais leur taille diminue (fig. $6, E$ ). Chez le $\delta$, les arêtes disparaissent au niveau de la bourse caudale.

Mâle: corps long de $4,7 \mathrm{~mm}$, large de $90 \mu$ dans sa partie moyenne, ailes comprises. Vésicule céphalique haute de $50 \mu$ sur $30 \mu$ de large. Anneau nerveux, pore excréteur et déirides situés respectivement à $160 \mu$, $122 \mu$ et $200 \mu$ de l'apex. Canal excréteur long de $60 \mu$. Esophage long de $270 \mu$. (fig. 6, A). Bourse caudale figurée en $6, J$. Papilles prébursales situées à $40 \mu$ en avant de la bourse caudale. Spicules ailés, longs de $180 \mu$, à extrémité distale bifide (fig. $6, K, L$ ). Gubernaculum haut de $110 \mu$ (fig. $6, O$ ). Cône génital avec papille 0 triangulaire (fig. $6, N$ ) et papilles 7 en forme de crochets (fig. $6, M$ ).

Femelle: corps long de 5,8 mm, large de $130 \mu$ dans sa partie moyenne, ailes comprises. Vésicule céphalique haute de $55 \mu$ sur $30 \mu$ de large. Anneau nerveux, pore excréteur et déirides situés respectivement à $128 \mu, 110 \mu$ et $225 \mu$ de l'apex. Canal excréteur long de $65 \mu$. Esophage long de $380 \mu$. Queue : $70 \mu$; filament caudal : $19 \mu$. La vulve s'ouvre à $1,1 \mathrm{~mm}$ de la pointe caudale. Didelphie. Vagina vera: $14 \mu$. Branche antérieure de l'ovéjecteur: vestibule, $80 \mu$; sphincter, $35 \mu$; trompe, $19 \mu$; branche postérieure de l'ovéjecteur: vestibule, $30 \mu$; sphincter, $25 \mu$; trompe, $16 \mu$ (fig. 6, G). Eufs hauts de $62 \mu$ sur $38 \mu$ de large.

\section{Discussion.}

Pour les raisons que nous exposerons plus loin (voir discussion sur Molinostrongylus bauchoti), nous considérons que cette espèce est nouvelle et nous la nommons Molinostrongylus benexae $\mathrm{n}$. sp., en la dédiant à notre collègue, $\mathbf{M}^{\mathrm{m} \bullet}$ Jacqueline Benex. 

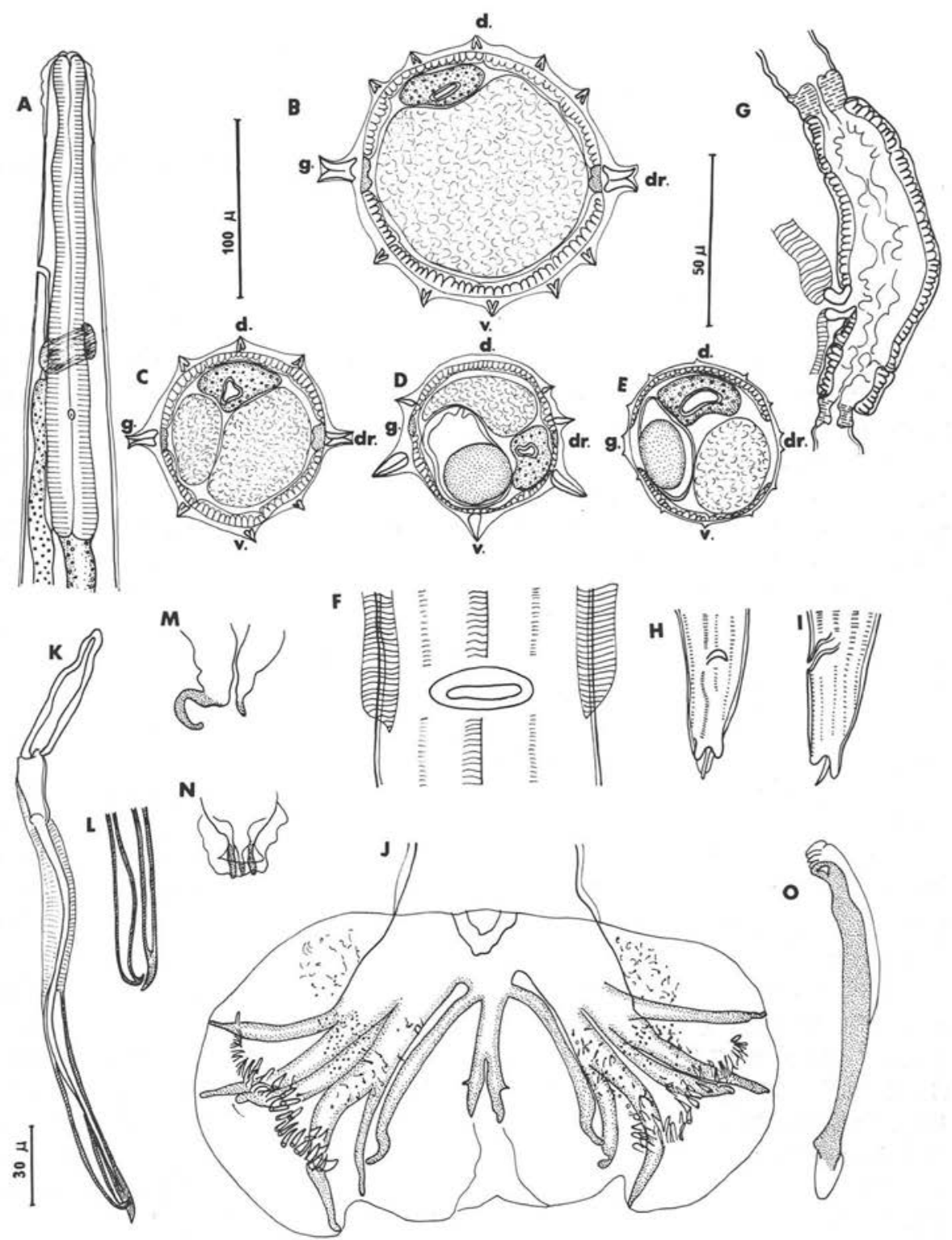

FIG. 6. - Molinostrongylus benexae n. sp. A : ô, extrémité antérieure, vue latérale gauche; B : coupe transversale au milieu du corps; C : + , id ; D : + , coupe transversale au-dessus du niveau de la vulve; E: + , coupe transversale entre les niveaux de la vulve et de l'anus; F : $q$, région vulvaire, détail des arêtes cuticulaires, vue ventrale; $\mathrm{G}: \mathcal{q}$, ovéjecteur, vue latérale gauche $; \mathrm{H}, \mathrm{I}: \stackrel{\circ}{+}$, extrémité caudale, vues ventrale et latérale gauche; $\mathrm{J}: \delta^{\star}$, bourse caudale, vue ventrale; $\mathrm{K}: \delta$, spicule gauche, vue ventrale; $\mathrm{L}: \delta$, pointes du spicule gauche disséqué (préparation écrasée); $\mathbf{M}, \mathbf{N}: \delta$. cône génital, vues latérale droite et ventrale; $\mathrm{O}: \delta^{2}$, gubernaculum, vue latérale droite.

A, C, D, E, F, G, H, I, J : éch. $100 \mu ; \mathrm{B}, \mathrm{M}, \mathrm{N}, \mathrm{O}$ : éch. $50 \mu ; \mathrm{K}, \mathrm{L}$ : éch. $30 \mu$. 


\section{Molinostrongylus bauchoti $\mathrm{n} . \mathrm{sp}$.}

MATÉRIEl TYPE: 1 \%, 1 partie postérieure de ơ, $\mathrm{n}^{\circ} 1078 \mathrm{~F}$.

Hôte : Chaerophon sp.

LOCALISATION : intestin.

ORIGINE GÉOGRAPHIQUE: Tananarive, Madagascar.

\section{DESCRIPTION.}

Le corps est déroulé. Comme chez les deux autres espèces malgaches, au moins chez la $q$ (la partie antérieure du $\sigma^{\star}$ n'étant pas connue), la vésicule céphalique est allongée, le pore excréteur situé antérieurement à l'anneau nerveux, le canal excréteur très long, le glandes excrétrices bien visibles et les déirides très postérieures (fig. $7, A$ ). La queue de la femelle porte trois pétales caudaux dont le dorsal est plus grand que les deux latéro-ventraux (fig. 7, E).

Synlophe: corps parcouru longitudinalement par 3 comarêtes ventrales, 3 comarêtes dorsales et 2 comarêtes latérales hautes de $18 \mu$ (ailes) (fig. 7, B). Chez la q, les ailes débutent au niveau du pore excréteur et les autres arêtes au niveau de l'anneau nerveux. Les ailes diminuent de taille au niveau de la vulve (fig. $7, C$ ) et disparaissent avec les autres arêtes entre la vulve et l'anus. Chez le $\delta$, les ailes et les arêtes disparaissent juste en avant de la bourse caudale.

Mâle: bourse caudale figurée en $7, F, G$. Spicules ailés, longs de $190 \mu$, bifides à leur extrémité postérieure. La branche interne du spicule est recourbée dorsalement (fig. 7, H). Gubernaculum haut de $85 \mu$. Cône génital portant sur sa lèvre postérieure deux papilles 7 allongées et sur sa lèvre antérieure la papille 0 arrondie (fig. $7, G$ ).

Femelle: corps long de $6,2 \mathrm{~mm}$, large de $95 \mu$. dans sa partie moyenne, ailes comprises. Vésicule céphalique haute de $70 \mu$ sur $33 \mu$ de large. Anneau nerveux, pore excréteur et déirides situés respectivement à $128 \mu$, $95 \mu$ et $310 \mu$ de l'apex. Canal excréteur long de $65 \mu$. Esophage long de $370 \mu$ (fig. 7, A). Queue : $80 \mu$; filament caudal : $16 \mu$ (fig. 7, E). La vulve s'ouvre à $1,6 \mathrm{~mm}$ de la pointe caudale. Didelphie. Vagina vera : $30 \mu$. Branche antérieure de l'ovéjecteur : vestibule, $50 \mu$; sphincter, $30 \mu$; trompe, $22 \mu$. Branche postérieure de l'ovéjecteur: vestibule, $40 \mu$; sphincter, $29 \mu$; trompe, $18 \mu$ (fig. $7, D)$. Nombreux œufs hauts de $68 \mu \times 40 \mu$.

\section{Discussion.}

Les trois espèces de Madagascar s'opposent à celles qui sont connues dans le reste du monde par le synlophe constitué, au moins chez la femelle, de deux ailes latérales et de six arêtes longitudinales.

Cette disposition se retrouve dans le groupe alatus, mais, dans ce dernier cas, les ailes sont plus grandes et les arêtes plus petites.

De plus, un ensemble de caractères ne se retrouve que chez les espèces malgaches : vésicule céphalique allongée, canal excréteur très long; déirides situées loin du pore 

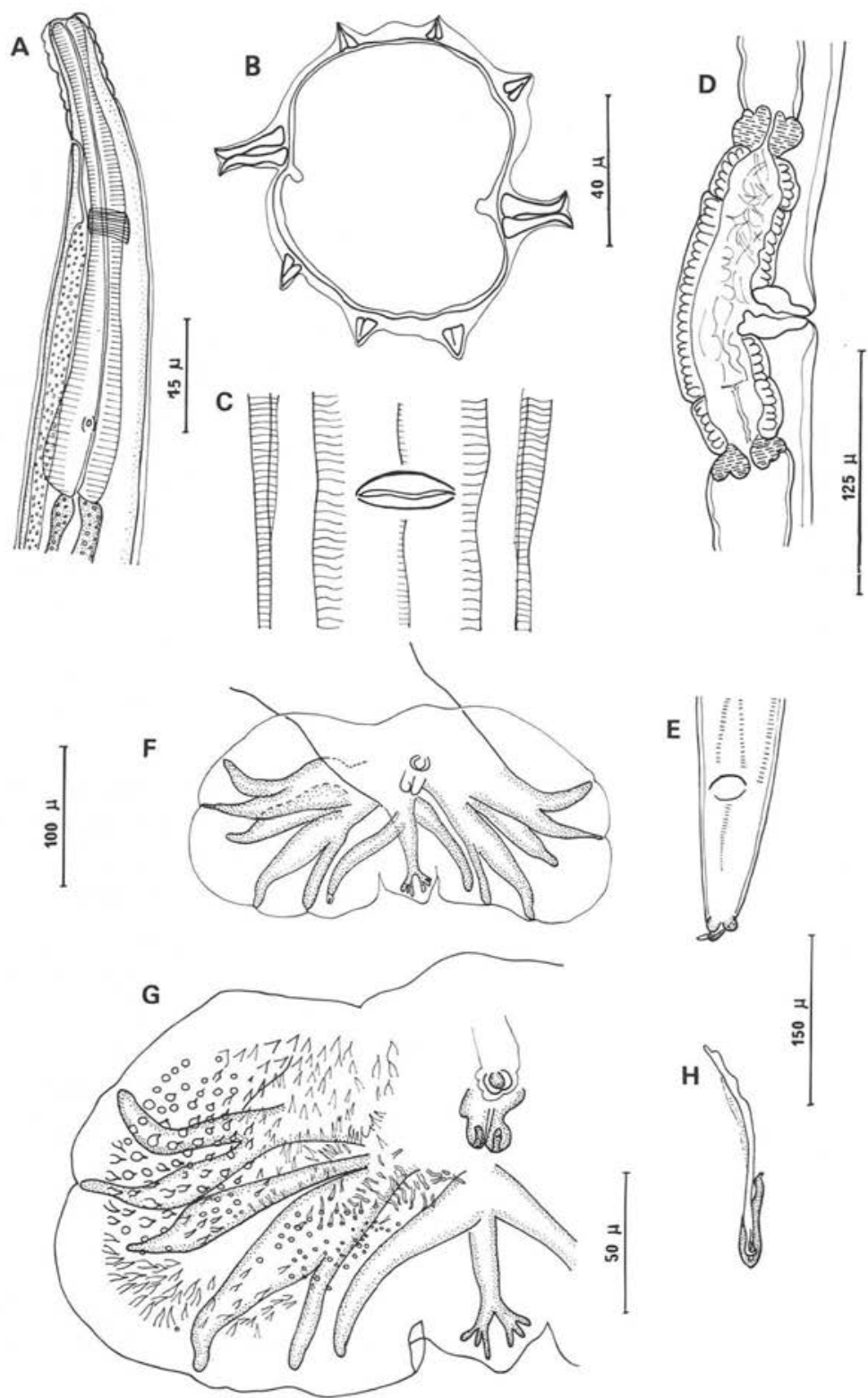

FIg. 7. - Molinostrongylus bauchoti n. sp. A : ơ, extrémité antérieure, vue latérale gauche ; B : ó, coupe transversale au milieu du corps; C: ${ }^{\text {, }}$, région vulvaire, détail des crêtes cuticulaires, vue ventrale; D : $\&$, ovéjecteur, vue latérale droite; E: + , extrémité postérieure, vue ventrale; F : $\delta^{\prime}$, bourse caudale (sans la spinulation), vue ventrale ; G : $\delta^{\star}$, lobe droit de la bourse caudale, détail de la spinulation, vue ventrale; $\mathrm{H}: \delta^{\pi}$, spicule gauche et gubernaculum, vue latérale gauche.

A : éch. $75 \mu$; B : éch. $40 \mu$; C, D, E : éch. $125 \mu$; F : éch. $100 \mu$; G : éch. $50 \mu$; H: éch. 150 . 
excréteur, pore excréteur situé entre le bord de la vésicule céphalique et l'anneau nerveux.

L'ensemble malgache constitue donc un groupe homogène, mais les trois espèces décrites ci-dessus sont faciles à reconnaître entre elles. $M$. benexae présente un dimorphisme sexuel sur le synlophe, les mâles ayant dix arêtes, alors que les femelles n'en ont que six. Les épines qui ornent la bourse caudale sont très hétérogènes: la zone périphérique comprend deux à trois rangées d'épines très puissantes, alors que les plus internes sont réduites et piliformes.

$M$. richardae se rapproche de $M$. benexae par l'aspect des terminaisons des côtes 6 et 8 , mais s'en éloigne par l'ornementation de la bourse caudale constituée d'épines de taille plus homogène et par la terminaison de la côte 4 éloignée de la côte 3 , alors que, chez $M$. benexae, la côte 4 se termine à proximité de la côte 3 .

Le spécimen décrit ci-dessus, contrairement aux deux autres espèces, a des côtes 6 et 8 parallèles, l'extrémité de la 6 restant éloignée de celle de la 8 . De plus, la côte 5 est très dilatée.

Nous pensons que cette espèce est nouvelle et nous la nommons Molinostrongylus bauchoti n. sp., en la dédiant à $\mathrm{M}$. Bauchot, qui l'a récoltée.

\title{
III. - Etude des Trichostrongyloidea parasites de Chiroptères orientaux et africains
}

\author{
Molinostrongylus colleyi n. sp.
}

MATÉriel TYPe: 2 o, 4 , $\mathrm{n}^{\circ} 83 \mathrm{KL}$.

HôTE : Tylonycteris pachypus (Temninck).

LOCALISATION : intestin.

ORIGINe GÉOGRAPHIQUe: Ulu Gombak, District de Selangor, Malaisie.

L'espèce a été trouvée chez deux autres Tylonycteris pachypus (62 KL et $92 \mathrm{KL}$ ) et chez quatre Tylonycteris robustula Thomas de la même région (73 à $75 \mathrm{KL}, 79 \mathrm{KL})$.

\section{DESCRIPTION.}

Le corps est déroulé. En vue apicale, la tête porte 2 amphides, 6 papilles labiales externes et 4 papilles céphaliques. Les trois lobes œsophagiens font saillie au-dessus de l'ouverture buccale (fig. $8, B$ ). Pore excréteur et déirides au même niveau, très postérieurs. Canal excréteur court. Euf gros. Queue de la femelle avec trois pétales caudaux, le dorsal étant le plus long (fig. $8, E$ ). Branche postérieure de l'ovéjecteur très réduite $(f i g .8, D)$. Ornementation pileuse de la bourse caudale réduite $(f i g .8, F)$.

Synlophe: chez les deux sexes, corps parcouru longitudinalement par deux ailes latérales qui débutent sur le bord postérieur de la vésicule céphalique et s'étendent jusqu'à environ $300 \mu$ en avant de la bourse caudale chez le $\delta^{*}$, et jusqu'au niveau 

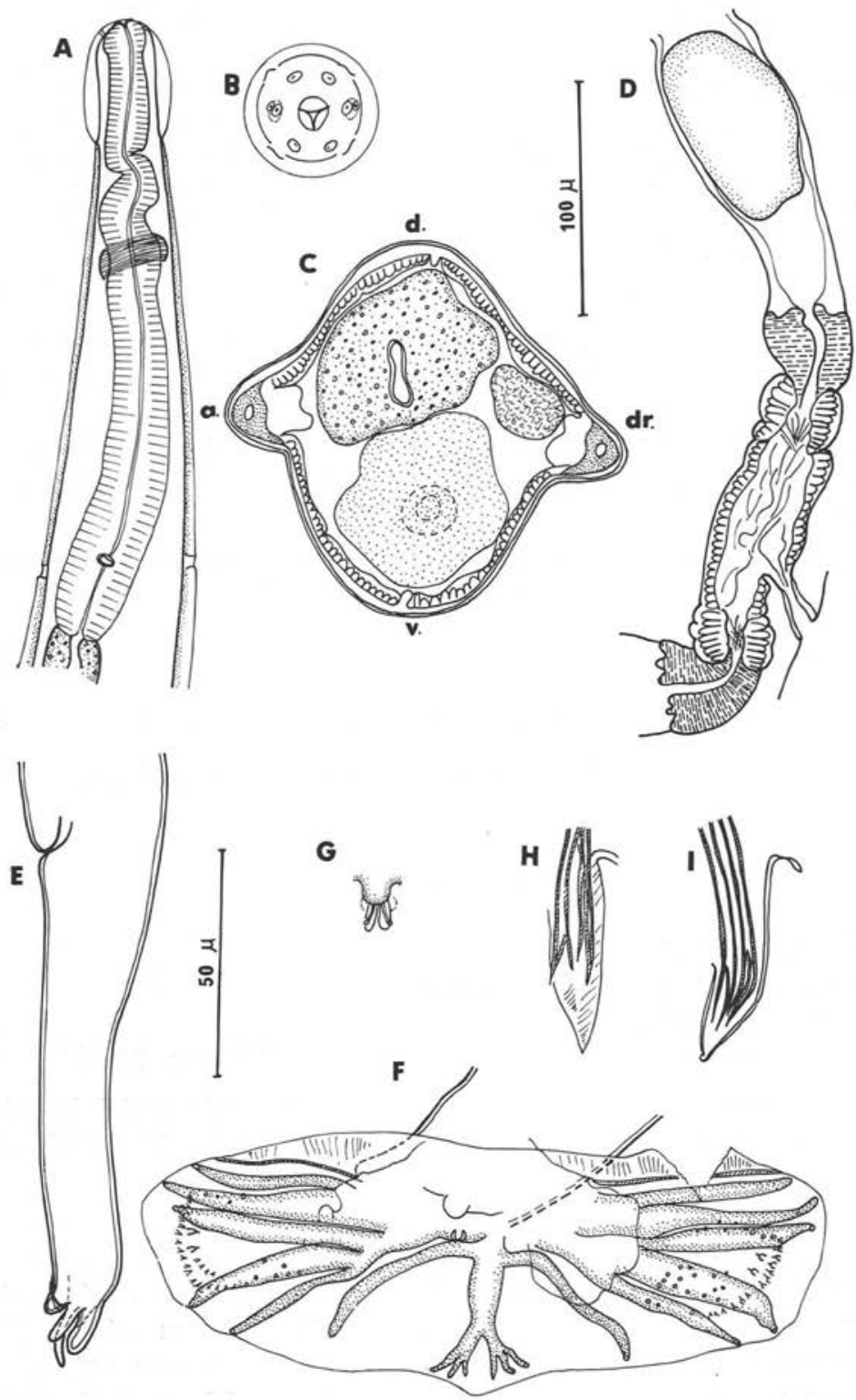

Fig. 8. -- Molinostrongylus colleyi n. sp. A : đ̋, extrémité antérieure, vue ventrale; B : tête en vue apicale; C: + , coupe transversale au milieu du corps; D: $q$, ovéjecteur, vue latérale droite ; E : + , extrémité postérieure, vue latérale gauche ; F : $\delta^{*}$, bourse caudale, vue ventrale; $\mathrm{G}: \delta^{+}$, cône génital; H, I : $\delta^{\text {, }}$ pointe des spicules et gubernaculum, vues ventrale et latérale gauche.

A, D, E : éch. $100 \mu$; B, C, F, G, H, I : éch. $50 \mu$. 
de la vulve chez la + . En coupe transversale, ces ailes se présentent sous forme d'une plaque peu saillante (fig. $8, C$ ).

Mâle: corps long de $4,2 \mathrm{~mm}$, large de $90 \mu$ dans sa partie moyenne, ailes comprises. Vésicule céphalique haute de $52 \mu \times 37 \mu$ de large. Anneau nerveux, pore excréteur et déirides situés respectivement à $95 \mu, 235 \mu, 230 \mu$ (déiride gauche), $240 \mu$. (déiride droite) de l'apex. Esophage long de $265 \mu$ (fig. 8, A). Bourse caudale figurée en $8, F$. Spicules ailés, longs de $160 \mu$, à extrémité distale bifide (fig. $8, I$ ). Gubernaculum haut de $58 \mu$ (fig. $8, H$ ). Cône génital peu développé, portant sur sa lèvre postérieure deux papilles 7 allongées (fig. $8, G$ ).

Femelle : corps long de $5,3 \mathrm{~mm}$, large de $90 \mu$ dans sa partie moyennes, ailes comprises. Vésicule céphalique haute de $35 \mu \times 31 \mu$. Anneau nerveux, pore excréteur et déirides situés respectivement à $130 \mu, 230 \mu, 240 \mu$ (déiride gauche), $250 \mu$ (déiride droite) de l'apex. Esophage long de $320 \mu$. Queue : $220 \mu$; filament caudal : $27 \mu$ (fig. $8, E$ ). La vulve s'ouvre à $1,2 \mathrm{~mm}$ de la pointe caudale. Didelphie. Vagina vera: $24 \mu$. Branche antérieure de l'ovéjecteur : vestibule, $54 \mu$; sphincter, $30 \mu$; trompe, $36 \mu$. Branche postérieure de l'ovéjecteur: vestibule, $25 \mu$; sphincter, $18 \mu$; trompe, $40 \mu$. (fig. 8, D). Utérus antérieur: $935 \mu$, avec 6 œufs; utérus postérieur : $365 \mu$, avec 4 œufs. Eufs hauts de $83 \mu$. sur $45 \mu$ de large.

\section{Discussion.}

M. vespertilionis Morosov et Spasski, 1961 est la seule espèce qui paraisse avoir des affinités avec celle qui est décrite ci-dessus, car les ailes latérales se présentent, en coupe transversale, sous forme d'une large plaque relativement peu saillante. La queue de la femelle, dans les deux cas, porte une pointe caudale beaucoup plus longue que les latérales. Les côtes 5 et 6 convergent relativement peu l'une vers l'autre et ne forment pas la «pince » que l'on observe chez la plupart des Molinostrongylus. Les deux espèces restent cependant faciles à distinguer, car les spicules se terminent par deux pointes, au lieu de trois chez $M$. vespertilionis, et la lame chitinoïde qui constitue l'armature des ailes latérales est plus arrondie que chez vespertilionis, où elle est presque plate. Nous considérons donc l'espèce comme nouvelle et nous la nommons Molinostrongylus colleyi, en la dédiant à M. F. Colley.

\section{Molinostrongylus owyangi n. sp.}

MATÉRIEL TYPe: 2 đ, 1 \%, $\mathrm{n}^{\circ} 61 \mathrm{KL}$.

Hôte: Glischropus tylopus (Dobson).

LOCALISATION : intestin.

OrIgINe GÉOGRAPHIQUe: Ulu Gombak, District de Selangor, Malaisie.

\section{DESCRIPTION.}

Le corps est légèrement courbé le long de la ligne ventrale. Anneau nerveux, pore excréteur et déirides au même niveau, situés à peu près à la moitié de l'œsophage. Ornementation pileuse de la bourse caudale réduite (fig. 9, F). Queue de la femelle avec trois pétales caudaux, le dorsal étant le plus long (fig. 9, D). 


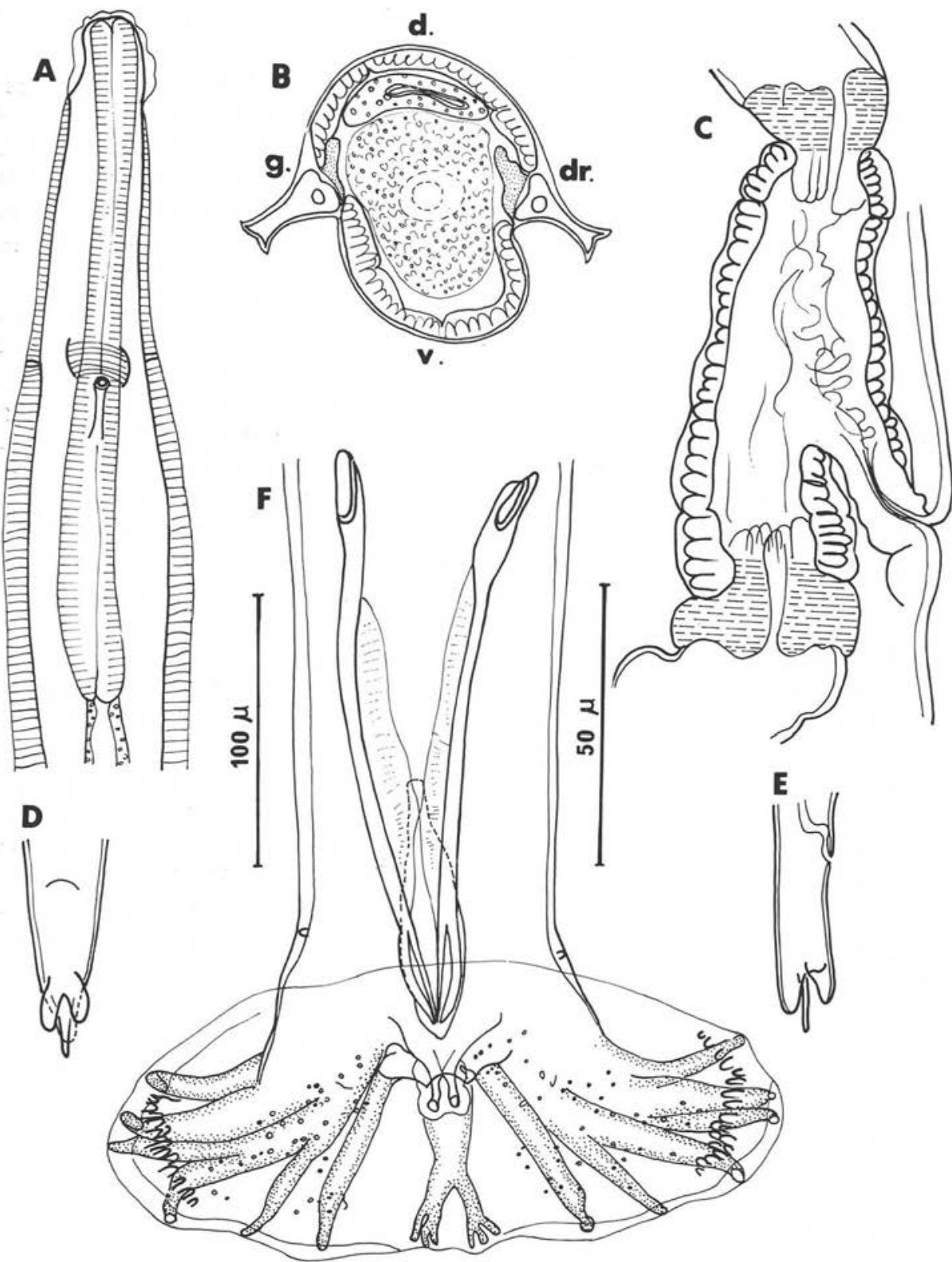

FIG. 9. - Molinostrongylus owyangi n. sp. A : $\$$, extrémité antérieure, vue ventrale; B : 9 , coupe transversale au milieu du corps; C: $q$, ovéjecteur, vue latérale droite; D, E : $q$, extrémité postérieure, vues ventrale et latérale droite; F : o , bourse caudale, vue ventrale. A, D, E: éch. $100 \mu$; B, C, F : éch. $50 \mu$. 
Synlophe: chez les deux sexes, corps parcouru longitudinalement par deux ailes latérales de 17 à $18 \mu$ de haut (fig. 9, B). Les ailes débutent sur le bord postérieur de la vésicule céphalique et s'étendent jusqu'à environ $50 \mu$ en avant de la bourse caudale chez le $\delta^{*}$, jusqu'à la queue chez la $q$.

Mâle: corps long de $1,85 \mathrm{~mm}$, large de $60 \mu$ dans sa partie moyenne, ailes comprises. Vésicule céphalique haute de $30 \mu$ sur $24 \mu$ de large. Anneau nerveux, pore excréteur et déirides situés respectivement à $118 \mu, 138 \mu$ et $140 \mu$ de l'apex. CEsophage long de $190 \mu$. Bourse caudale figurée en 9, F. Spicules ailés, longs de $110 \mu$, à extrémité distale bifide. Gubernaculum haut de $47 \mu$. Cône génital bien marqué. Papille 0 triangulaire, papilles 7 allongées (fig. 9, F).

Femelle immature: corps long de $2,75 \mathrm{~mm}$, large de $60 \mu$ dans sa partie moyenne, ailes comprises. Vésicule céphalique haute de $32 \mu \times 28 \mu$. Anneau nerveux, pore excréteur et déirides situés respectivement à $120 \mu$, $132 \mu$ et $122 \mu$ de l'apex. Esophage long de $245 \mu$ (fig. 9, A). Queue : $60 \mu$; filament caudal : $20 \mu$ (fig. 9, D, E). La vulve s'ouvre à $575 \mu$ de la pointe caudale. Didelphie. Vagina vera: $18 \mu$. Branche antérieure de l'ovéjecteur: vestibule, $37 \mu$; sphincter, $14 \mu$; trompe, $14 \mu$. Branche postérieure de l'ovéjecteur: vestibule, $17 \mu$; sphincter, $16 \mu$; trompe, $15 \mu$ (fig. 9, C).

Discussion.

L'espèce se rattache au groupe skrjabini, car le synlophe n'est constitué que par les deux ailes latérales. Elle se distingue des espèces de ce groupe par des spicules munis chacun de deux pointes terminales au lieu d'une. La taille des spicules est particulièrement réduite $(110 \mu)$.

Il s'agit donc d'une espèce nouvelle que nous nommons Molinostrongylus owyangi n. sp., en la dédiant à M. le $\mathrm{D}^{\mathrm{r}} \mathrm{C}$. K. Ow Yang.

\section{Allintoschius dunni n. sp.}

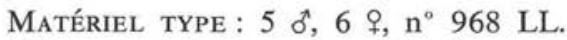

Hôte : Myotis mystacinus (Kuhl).

LOCALISATION : intestin.

Origine GÉographiQue: Bukit Mangol, Province de Selangor, Malaisie.

Autre MATÉrIel: Quelques spécimens chez cinq Pipistrellus nanus originaires de la Maboké, République Centrafricaine.

\section{DESCRIPTION.}

Le corps est enroulé de façon senestre le long de la ligne ventrale. En vue apicale, la tête porte 2 amphides, 6 papilles labiales externes et 4 papilles céphaliques. La bouche, de forme losangique, est entourée par quatre pétales arrondis (fig. 10, B).

Synlophe: dans les deux sexes, le corps est parcouru longitudinalement par $25\left(0^{*}\right)$ à 28 ( $($ ) crêtes cuticulaires qui débutent soit à environ $80 \mu$ en arrière de la vésicule céphalique (ventrales et dorsales), soit à différents niveaux le long des champs latéraux (fig. $10, E$ ). Les crêtes disparaissent en avant de la bourse caudale chez le $\tilde{\text {. }}$. 


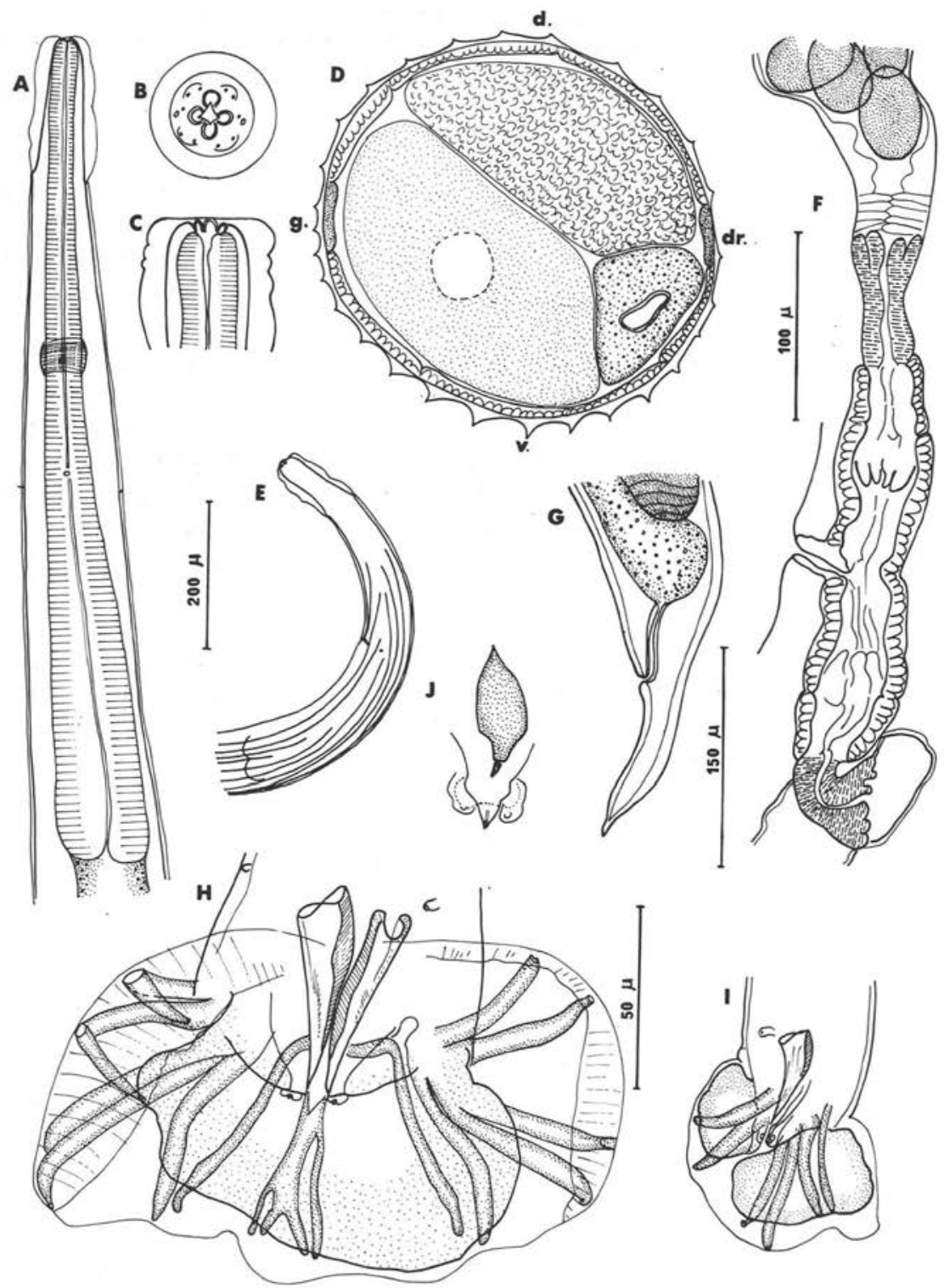

Fig. 10. - Allintoschius dunni n.sp. A: $\delta^{\star}$, extrémité antérieure, vue ventrale; B : q, tête en vue apicale; $\mathrm{C}: \mathcal{q}$, tête en vue ventrale; D: $q$, coupe transversale au milieu du corps; E : ô, extrémité antérieure, vue latérale gauche; F: $ᄋ$, ovéjecteur, vue latérale gauche; G : + , extrémité postérieure, vue latérale gauche; $\mathrm{H}: \delta$, bourse caudale, vue ventrale; I: $i d$., vue latérale gauche; $\mathbf{J}: \delta$, cône génital et gubernaculum, vue ventrale.

A, G: éch. $150 \mu ; \mathrm{B}, \mathrm{C}, \mathrm{D}, \mathrm{H}, \mathrm{J}$ : éch. $50 \mu ; \mathrm{F}, \mathrm{I}$ : éch. $100 \mu ; \mathrm{E}$ : éch. $200 \mu$. 
au niveau de l'anus chez la ․ En coupe transversale, la pointe des crêtes est orientée de la droite vers la gauche pour les deux faces. Les crêtes ventrales sont les plus développées (fig. 10,D). Ce synlophe est identique à celui du genre didelphe Citellinema Hall, 1916.

Mâle: corps long de $4,3 \mathrm{~mm}$, large de $80 \mu$ dans sa partie moyenne. Vésicule céphalique haute de $95 \mu \times 40 \mu$ de large. Anneau nerveux, pore excréteur et déirides situés respectivement à $205 \mu, 300 \mu$ et $310 \mu$. de l'apex. Esophage long de $565 \mu$. Bourse caudale figurée en $10, H, I$. Présence d'une dilatation cuticulaire à l'intérieur de la bourse caudale, constituant une sorte de sphère, avec invagination en entonnoir aboutissant au cloaque. Les parois de la bourse sont appliquées sur cette sphère et ne peuvent être mises à plat, en vue ventrale. L'asymétrie de la dorsale et des externodorsales se retrouve sur tous les spécimens examinés, avec une disposition comparable à celle qui est figurée. Spicules de forme triangulaire, ailés, hauts de $70 \mu$ (fig. 10, H) . Cône génital bien développé, portant la papille 0 allongée et des papilles 7 en forme de pétales (fig. 10, J).

Femelle: corps long de $8 \mathrm{~mm}$, large de $100 \mu$ dans sa partie moyenne. Vésicule céphalique haute de $110 \mu$ sur $50 \mu$ de large. Anneau nerveux, pore excréteur et déirides situés respectivement à $180 \mu, 320 \mu$ et $335 \mu$ de l'apex. Esophage long de $510 \mu$ (fig. 10, A). Queue effilée, longue de $110 \mu$ (fig. 10, G). La vulve s'ouvre à $1,7 \mathrm{~mm}$ de la pointe caudale. Didelphie. Vagina vera: $27 \mu$. Branche antérieure de l'ovéjecteur: vestibule, $80 \mu$; sphincter, $32 \mu$; trompe, $72 \mu$. Branche postérieure de l'ovéjecteur: vestibule, $63 \mu$; sphincter, $35 \mu$; trompe, $60 \mu$. La branche utérine antérieure mesure $1,9 \mathrm{~mm}$, postérieure $1,15 \mathrm{~mm}$. Les branches utérines contiennent de nombreux œufs hauts de $50 \mu$ sur $33 \mu$ de large (fig. 10, F).

\section{Discussion.}

L'espèce type d'Allintoschius Chitwood, 1937 est pourvue de très petits tubercules près de la pointe caudale de la femelle, du même type que ceux du genre Molineus. L'espèce type de Parallintoschius Araujo, 1940 a de gros tubercules et une pointe caudale du même type que ceux du genre Anoplostrongylus. Les deux genres ont donc souvent été classés dans des sous-familles différentes, mais cette solution n'est pas acceptable, car l'on connaît maintenant d'assez nombreuses espèces ayant des pointes caudales femelles très variées: pointues, avec épines, avec épines et tubercules, alors que l'ensemble est très homogène et bien caractéristique. Il est manifeste ici, comme dans d'autres genres (Molineus, par exemple), que la pointe caudale femelle est un bon caractère, mais ne peut suffire à elle seule pour séparer les Anoplostrongylinae des Molineinae. La limite entre ces deux sous-familles, si elle existe, ne pourra être donnée qu'en s'appuyant également sur les caractères fournis par le synlophe.

Nous pensons donc que le genre Parallintoschius doit être placé en synonymie du genre Allintoschius.

L'espèce du Myotis paraît caractérisée par l'existence des quatre petits pétales labiaux, mais ceux-ci sont tellement réduits qu'ils peuvent échapper à l'observation. 
Deux espèces sont particulièrement proches, par la queue de la femelle, la forme de la bourse caudale, l'aspect des spicules et la vulve dépourvue de lèvres saillantes. Ce sont A. tadaridae (Caballero, 1942) n. cb. et A. nudicaudus (Freitas et Machado, 1963) n. cb. La distinction entre les espèces peut se faire sur la forme de la dorsale et des externo-dorsales ; l'asymétrie de la bourse caudale de la forme du Vieux Monde, bien que légère, est cependant plus marquée que chez les deux espèces connues du Nouveau Monde.

Nous pensons donc que l'espèce est nouvelle et nous la nommons Allintoschius dunni $\mathrm{n}$. sp., en la dédiant au $\mathrm{D}^{r}$ Dunn.

Considérant que l'attribution de l'espèce Anoplostrongylus delicatus Schwartz, 1927 au genre Molinostrongylus était erronée, on pouvait constater jusqu'à maintenant que chacun des genres de Trichostrongyloidea parasites de Chiroptères était caractéristique soit de l'Ancien Monde, soit du Nouveau Monde. Dans le cas présent, l'espèce est indiscutablement très proche des formes américaines, et le genre Allinstoschius est donc le seul qui ait une répartition aussi étendue.

\section{IV. - Considérations sur le genre Molinostrongylus}

Le genre Molinostrongylus a longtemps été confondu avec Anoplostrongylus Boulenger, 1926 et en a été séparé par Skarbilovitsch (1934), qui s'est appuyé principalement sur les caractères des spicules, de la côte dorsale et de l'extrémité caudale de la femelle.

Nous avons indiqué précédemment (cf. Durette-Desset et Chabaud, 1975) que les Anoplostrongylinae caractéristiques du Vieux Monde (Molinostrongylus, Nycteridostrongylus) se distinguent de ceux qui sont typiquement du Nouveau Monde par l'ornementation épineuse de la face ventrale de la bourse caudale et surtout par la côte 4 , qui se termine tout près de la côte 3 , et non à mi-distance entre la 3 et la 5 .

Anoplostrongylus delicatus Schwartz, 1928, bien que n'ayant pas le capuchon céphalique de l'espèce type, est donc maintenu dans le genre Anoplostrongylus, ainsi qu'il a été admis par Barus et Rysavy, 1971.

Le genre Molinostrongylus, remarquablement homogène, compte de nombreuses espèces qui sont cependant généralement faciles à distinguer les unes des autres, si l'on s'appuie sur la disposition des côtes de la bourse caudale, la pointe des spicules et les caractères du synlophe. La bibliographie présente cependant de nombreuses difficultés, car les bourses caudales ne sont pas toujours figurées étalées en vue ventrale, la forme de la pointe des spicules n'est réellement appréciable qu'après dissection de ceux-ci, et le synlophe est généralement peu décrit et non figuré.

Ces réserves étant faites, il semble cependant que les espèces peuvent se répartir en cinq groupes. Il est remarquable de constater que chacun de ces groupes, fondé 
avant tout sur les caractères du synlophe, semble correspondre à un genre bien déterminé de Chiroptères. Les statistiques faites par Andreiko et coll. (1968), par exemple, semblent indiquer la présence presque constante d'une espèce déterminée chez un Chiroptère déterminé, mais beaucoup d'autres Chiroptères sont parasités par cette même espèce avec des pourcentages de fréquence non négligeables. En France également, Strongylacantha glycirrhiza est très caractéristique des Rhinolophoidea, mais se trouve avec une certaine fréquence chez Miniopterus schrebersi, qui vit dans les mêmes grottes. Il est étrange que ces parasitismes accidentels, si fréquents depuis des millénaires, n'aient pas abouti à une véritable adaptation, et que la spécificité parasitaire reste bien marquée: on remarquera en effet que, sur les 14 espèces de Chiroptères qui nous ont été fournies par le $\mathrm{P}^{\mathrm{r}}$ Aellen, nous avons trouvé une faune très diversifiée, mais relativement bien caractéristique de chaque espèce (cf. tableau).

Dans le genre Molinostrongylus, les cinq groupes qui nous paraissent pouvoir être individualisés sont les suivants :

\section{1) Groupe ornatus.}

Synlophe au milieu du corps : 2 ailes latérales, 7 arêtes ventrales, 7 arêtes dorsales. Côte dorsale courte ou très courte, se terminant par deux rameaux bifides. Pointe des spicules double ou triple. Parasites des Miniopterinae.

Le groupe comprend six espèces: $M$. ornatus (Mönnig, 1927), d'Afrique du Sud ; M. pseudornatus Yeh, 1957, de Rhodésie ; M. panousei Dollfus, 1954, d'Afrique du Nord, de France et de la région méditerranéenne jusqu'à l'Afghanistan; $M$. dollfusi Thomas, 1958 et $M$. heydoni (Baylis, 1930), d'Australie ; M. rhinolophi Yamaguti, 1941, du Japon. Bien que décrite chez un Rhinolophe, cette dernière espèce a été retrouvée récemment chez un Minioptère par Kagei et Sawada (1973).

\section{2) Groupe richardae.}

Synlophe au milieu du corps : 2 ailes latérales, 3 grosses arêtes ventrales, 3 grosses arêtes dorsales ( 5 chez le mâle de $M$. benexae). Côte dorsale courte, se terminant par deux rameaux bi ou trifides. Pointe des spicules double. Parasites de Molossidae malgaches.

Le genre comprend trois espèces: $M$. richardae n. sp.; $M$. bauchoti n. sp.; $M$. benexae $\mathrm{n} . \mathrm{sp}$.

\section{3) Groupe alatus.}

Synlophe au milieu du corps : 2 ailes latérales, 3 petites arêtes ventrales, 3 petites arêtes dorsales. Côte dorsale longue ou très longue, se terminant par deux rameaux trifides. Spicules très longs, pointe des spicules double. Parasites du genre Myotis d'Afrique et d'Europe.

Le groupe ne comprend que l'espèce $M$. alatus (Ortlepp, 1932), mais il n'est pas impossible que $M$. spasskii Andreiko, Pinchuk et Skvorzov, 1968 dont daubentonii Zdzitowiecki, 1970 est peut-être synonyme, également parasite de Myotis, ait un synlophe du même type et appartienne au même groupe. 


\title{
4) Groupe skrjabini.
}

Synlophe au milieu du corps : 2 ailes latérales saillantes; absence d'arêtes dorsales et ventrales. Côte dorsale de longueur moyenne, se terminant par deux rameaux bi ou trifides. Pointe des spicules simple ou double. Parasites du genre Nyctalus (et Glischropus, qui en est proche) des régions paléarctique et orientale.

Le groupe comprend quatre espèces: M. skrjabini Skarbilovitsch, 1934, parasite de Nyctalus noctula; $M$. aelleni n. sp., parasite de $N$. lasiopterus; $M$. longispiculus (Yamashita et Mori, 1953), parasite de $N$. maximus; $M$. owyangi n. sp., parasite de Glischropus tylopus. Nous avons vu plus haut qu'une autre espèce non déterminée du même groupe parasitait $N$. leisleri.

\section{5) Groupe vespertilionis.}

Synlophe au milieu du corps: deux formations latérales formant des ailes larges et plates, absence d'arêtes dorsales et ventrales. Côte dorsale de longueur moyenne, se terminant par deux rameaux bifides. Pointe des spicules avec deux ou trois terminaisons. Parasites de Pipistrellus en Europe et de Tylonycteris en Malaisie.

Le groupe comprend deux espèces: $M$. vespertilionis Morosov et Spasski, 1969, parasite de Pipistrellus; $M$. colleyi n. sp., parasite de Tylonycteris.

M. morosovi, Andreiko et coll., 1968 (= M. skrjabini sensu Morozov et Spasski, 1961), n'est pas pris en considération, car, s'il est exact que le dessin des pointes des spicules et la terminaison de la dorsale donnée par Morozov et Spasski ne correspondent pas à skrjabini, ces éléments sont tout à fait insuffisants pour caractériser une espèce, d'autant plus que quatre hôtes différents sont cités.

En conclusion, les 17 espèces qui nous paraissent actuellement valides se répartissent, d'après la constitution du synlophe, en cinq groupes distincts, paraissant correspondre chacun à un hôte ou à un groupe d'hôtes bien déterminé. Il est difficile d'affirmer dans quel sens s'est effectuée l'évolution. Nous pensons personnellement qu'elle s'est faite dans le sens d'une atrophie progressive du synlophe.

\section{V. - Composition de la Nematofaune (Trichostrongyloidea) des Chiroptères}

\author{
Hypothèses sur sa formation
}

Barus et Rysavy (1971) se sont récemment intéressés à ce problème et ont publié une revue très utile à ce sujet. Des éléments nouveaux concernant, d'une part, les Nématodes de Tupaia, d'autre part, la présence d'une espèce typiquement américaine dans le Vieux Monde, et enfin la connaissance du synlophe d'un certain nombre d'espèces, nous amènent cependant à reprendre leur travail pour aboutir à des conclusions partiellement différentes. 
Les Trichostrongyloïdes de Chiroptères nous paraissent pouvoir se scinder en six groupes :

Groupe I : Strongylacantha Beneden, 1873.

Le genre, comprenant trois espèces du Vieux Monde, a une capsule buccale puissamment armée (fig. 11, $A$ à $C$ ), d'un type très primitif, et constitue un groupe parfaitement autonome, qui nous paraît n'avoir aucun rapport direct avec le groupe IV. Bien que se rencontrant accidentellement chez de nombreuses chauves-souris, ce groupe est très caractéristique des Rhinolophoïdes, que les Mammalogistes considèrent comme les plus primitifs des Microchiroptères (cf. Brosset, 1966). S. glycirrhiza, tout au moins, n'a pas de synlophe.

Groupe II : Nématodes du Tupaia; Anoplostrongylus Boulenger, 1926; Nycteridostrongylus Baylis, 1930 ; Allintoschius Chitwood, 1937.

En dehors du groupe Strongylacantha, les genres qui nous paraissent les plus primitifs chez les chauves-souris sont Anoplostrongylus et Nycteridostrongylus. Cette idée nous semble renforcée du fait que ce sont précisément ces deux genres que l'on trouve chez les Tupaiidae.

a) FAUNE DU Tupaia.

Nous rappelons ici que les trois Trichostrongylides découverts chez les Tupaia en Malaisie sont les suivants :

1) Hepatojarakus malayae Yeh, 1955 est un Molineinae évolué : corps dilaté avec synlophe formé de nombreuses crénelures et bourse caudale évoquant celle du genre Molinostrongylus.

2) Nycteridostrongylus (Petiellus) petersi Durette-Desset et Chabaud, 1975 constitue un sous-genre de Nycteridostrongylus. Le sous-genre tautonyme est parasite de Chiroptères en Australie et au Viêt-nam. Le synlophe, très original, confirme totalement l'affinité des deux sous-genres.

3) Anoplostrongylus (Tupaiostrongylus) liei (Dunn, 1963) constitue un sous-genre d'Anoplostrongylus. Le sous-genre tautonyme est parasite de Chiroptères américains et n'a jamais été signalé dans l'Ancien Monde. Les affinités indiscutables entre Nématodes de Chiroptères et de Tupaia paraissent indiquer une origine commune aux deux groupes d'hôtes. La faune en Trichostrongyloidea semble s'être constituée à l'époque où ces hôtes se sont diversifiés, à partir de Nématodes Molineinae proches de ceux que l'on connaît actuellement chez les Insectivores très primitifs (Tenrecoidea) et les Carnivores.

b) GenRe Anoplostrongylus.

L'espèce type a un capuchon céphalique élaboré qui la relie au groupe III, alors que les deux autres espèces, rattachées au même genre, ont un capuchon céphalique simple. Nous préférons cependant nous rallier à l'avis de Barus et Rysavy, pour ne 

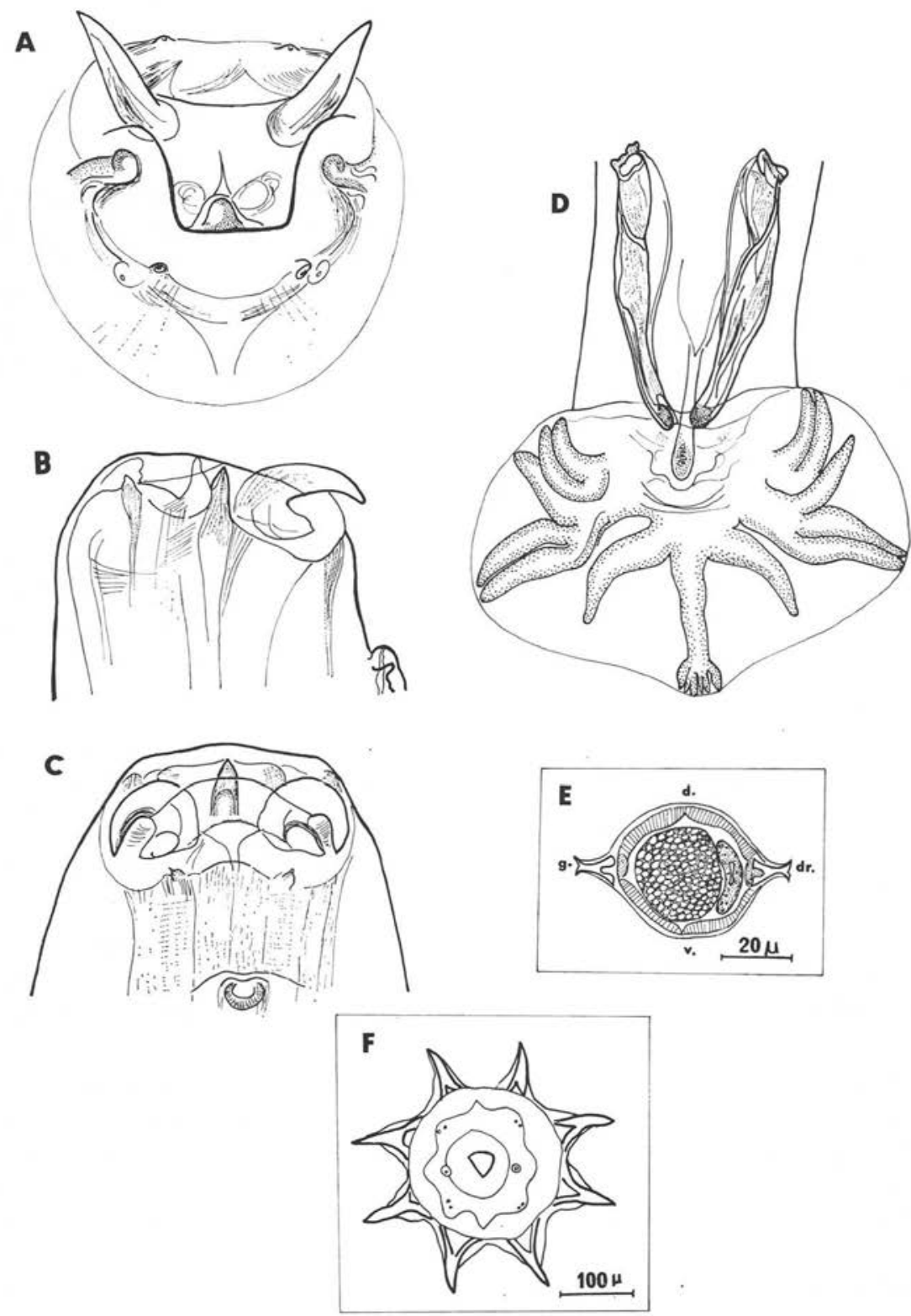

Fig. 11. - Strongylacantha glycirrhiza Van Beneden, 1873. A, B, C: tête en vues apicale, latérale droite et ventrale; D : $\delta^{2}$, bourse caudale, vue ventrale. - Molinostrongylus sp., chez Pipistrellus nathusii de Suisse. E: + , coupe transversale au milieu du corps. - Histiostrongylus coronatus Molin, 1861, chez Phyllostomus discolor au Venezuela (coll. Diaz-Ungria). $\mathrm{F}: \delta$, tête en vue apicale.

(A-D : d'après Desportes, dessins inédits Faune de France). 
pas multiplier abusivement les genres, et nous définissons le genre Anoplostrongylus par les trois fortes pointes caudales de la femelle et la disposition des côtes bursales (dorsale courte, pointes des côtes 2, 3, 4, 5, 6 écartées en éventail régulier, côtes 8 naissant à la base de la dorsale).

Le seul synlophe que nous connaissions actuellement est celui de l'espèce du Tupaia; il est très proche de celui des Molineus.

\section{c) GenRe Nycteridostrongylus.}

Le sous-genre du Tupaia et celui des Microchiroptères sont proches l'un de l'autre. Les bourses caudales, avec la terminaison de la côte 4 , proche de la 3 et éloignée de la 5 , sont déjà orientées vers les types de l'Ancien Monde. Le synlophe, très spécialisé, semble indiquer une petite lignée indépendante, issue des Molineinae et réduite à l'état de relique.

\section{d) Genre Allintoschius.}

Nous avons vu plus haut que nous considérons Parallintoschius Araujo, 1940 comme synonyme. Le genre comprend donc huit espèces, dont sept du Nouveau Monde. La nouvelle espèce décrite ici, trouvée en Afrique et en Malaisie, semble indiquer que le genre est effectivement très ancien, puisque c'est le seul de tous les Trichostrongyloïdes qui soit connu chez les Chiroptères de l'Ancien et du Nouveau Monde. La bourse caudale, avec la disposition 2-3 groupées, 4-5 groupées, 6 isolée, l'éloigne du type le plus primitif 2-3 groupées, 4 isolée, 5-6 groupées, que l'on rencontre chez les Molineus. Le synlophe ressemble étonnamment à celui de Citellinema (Molineinae). Il semble donc s'agir d'un genre ancien, ayant évolué pour son propre compte en lignée distincte.

Les genres groupés dans ce chapitre constituent donc un ensemble qui nous paraît très archaïque, du fait que leur morphologie reste très proche de celle des Molineus, chacun d'eux ayant évolué dans des voies divergentes. La presque totalité des espèces actuelles se trouve en Amérique du Sud, mais, pour tous les genres, il existe des vestiges de leur présence dans l'Ancien Monde, soit chez le Tupaia, soit chez certains Chiroptères eux-mêmes.

Nous supposons donc que l'origine de ces genres pourrait être située avant la séparation des différents continents austraux.

\section{Groupe III : Genres américains à capuchon céphalique.}

Les genres Torrestrongylus Vigueras, 1935 et Tricholeiperia Travassos, 1935 paraissent se rattacher directement au genre Anoplostrongylus et évoluer par formation d'un appareil de fixation constitué aux dépens de la vésicule céphalique. L'étude approfondie du synlophe serait donc particulièrement intéressante, d'une part, pour vérifier l'origine du groupe, d'autre part, pour chercher à savoir si l'acquisition de l'appareil de fixation céphalique entraîne ou non une atrophie du synlophe. 


\section{Groupe IV : Genres à tête armée.}

Nous admettons dans ce groupe quatre genres, deux du Nouveau Monde: Biacantha Wolfgang, 1954 et Histiostrongylus Molin, 1861, et deux de l'Ancien Monde: Neohistiostrongylus Barus et Rysavy, 1971 et Spinostrongylus Travassos, 1935. L'étude de la structure céphalique d'Histiostrongylus (fig. 11, F) nous conduit à considérer les crochets comme une élaboration secondaire du capuchon céphalique, n'ayant aucun rapport vrai avec les crochets buccaux de Strongylacantha. Cet appareil de fixation constitue une convergence avec celui de certains Oxyures (Cephaluris, etc.) ou avec celui de certains Spirurides (Histiocephalinae à partir des Habronèmes, ou Schistorophinae à partir des Acuarides).

Nous pensons donc qu'il s'agit d'élaborations progressives d'un appareil de fixation, qui joue le rôle de synlophe, et que les formes ayant peu de crochets (Biacantha) sont plus primitives que les formes ayant une armature très complexe (Spinostrongylus).

L'origine de ce groupe est difficile à déterminer. Il pourrait s'être constitué à partir d'ancêtres ayant un capuchon céphalique comparable à celui des Tricholeiperia.

Il est difficile de savoir s'il y a eu un ancêtre commun entre les quatre genres, ou s'il s'agit de convergence entre la faune des deux Mondes.

Les synlophes restent mal connus. Ils semblent persister chez les deux genres les plus primitifs: Biacantha dans le Nouveau Monde et Neohistiostrongylus dans l'Ancien Monde, et ont disparu chez les deux genres les plus évolués : Histiostrongylus du Nouveau Monde et Spinostrongylus de l'Ancien Monde.

\section{Groupe V: Genres de Chiroptères frugivores.}

Les genres Chiropteronema Sandground, 1929 et Bidigiticauda Chitwood, 1938 semblent pouvoir être groupés, car ils présentent tous deux une atrophie de la bourse caudale, qui évoque celle des Métastrongylides. Leur origine pourrait être précisée, si l'on connaissait leur synlophe. Le plus vraisemblable actuellement est de supposer qu'ils dérivent d'ancêtres proches d'Anoplostrongylus.

Le passage brutal du biotope d'un intestin d'Insectivore à celui d'un Frugivore paraît avoir entraîné des modifications importantes, qui convergent avec celles subies par les Métastrongylides dans le poumon.

\section{Groupe VI : Molinostrongylus. Genres proches, parasites de Pholidotes, Pri- mates et Sciuridés.}

Le genre Molinostrongylus a été analysé plus haut. Peu différent du genre Anoplostrongylus par l'ensemble de ses caractères, la bourse caudale est cependant bien caractéristique et le genre constitue donc la réussite évolutive de la lignée dans l'Ancien Monde. Alors que tous les Nématodes précédents sont très caractéristiques des Tupaia ou des Chiroptères et ne semblent pas avoir évolué plus loin, le genre Molinostrongylus, 
au contraire, semble s'être adapté à différents Mammifères de l'Ancien Monde et avoir donné naissance ou, plus vraisemblablement, avoir une origine commune avec cinq genres: Trichochenia Kou, 1958 = Manistrongylus Baer, 1959, = Pholidostrongylus Baer, 1959, parasite des Pholidotes; Pithecostrongylus Lubimov, 1930 ; Hooperstrongylus Lie et Ow Yang, 1963 ; Dollfustrongylus Quentin, 1970, parasites de Primates et de Sciuridés ; Angulocirrus Biocca et Le Roux, 1957, parasite de l'Oryctérope.

Nous avons pu constater que le synlophe de ces genres est pratiquement identique à celui du genre Molinostrongylus; la bourse caudale, de type Molineus (2-3 groupées, 4 isolée, 5-6 groupées), tend parfois vers le type Molinostrongylus (3-4 groupées). Les écailles ou les épines ornant la face ventrale de la bourse caudale évoquent également souvent celles des Molinostrongylus.

\section{Conclusion}

Nous avons tenté sur la figure 12 de résumer les hypothèses exposées dans le chapitre précédent. En résumé, le peuplement des Microchiroptères nous paraît être constitué de deux ensembles très différents: premièrement, et à lui seul, le genre Strongylacantha, très archaïque et inféodé au groupe le plus primitif de Microchiroptères, les Rhinolophoïdea de l'Ancien Monde ; deuxièmement, tout un ensemble de genres dérivant plus ou moins directement des Molineus (donc de Trichostrongyloïdes. d'Insectivores primitifs ou de Créondontes-Carnivores). A la base se situe un groupe de genres dont certains ont évolué de façon divergente et qui se trouvent aussi bien dans l'Ancien Monde que dans le Nouveau Monde, soit chez les Tupaia, soit chez les Chiroptères. Parmi ceux-ci, le genre Anoplostrongylus reste le moins spécialisé, et c'est à partir de lui, nous semble-t-il, que se sont différenciés deux grands groupes relativement plus modernes.

Dans le Nouveau Monde, toute une série de genres qui paraissent acquérir un appareil de fixation original par modification de la vésicule céphalique ou qui subissent des modifications brutales par adaptation à des Microchiroptères frugivores ; dans l'Ancien Monde, au contraire, un genre riche en espèces, beaucoup plus homogène, Molinostrongylus. L'origine de deux genres à tête armée de l'Ancien Monde reste difficile à situer.

Le fait que tous les genres relativement plus modernes soient différents dans l'Ancien et le Nouveau Monde implique que les Nématodes n'ont pas suivi leurs hôtes au cours des migrations par le détroit de Behring. Ce fait surprenant est rare, mais ne semble pas unique. Une interprétation comparable a été donnée pour tenter d'expliquer la répartition des Héligmosomes de Sciuridés (Durette-Desset, 1971).

En dehors du cas des Strongylacantha adaptés aux Rhinolophoidea, nous ne notons aucun phénomène d'évolution parallèle entre l'hôte et le parasite. Les Phyllostomatoidea, par exemple, ne paraissent pas avoir de parasites plus primitifs 
que ceux des Vespertilionoidea. Tout se passe comme si, excepté les Rhinolophoidea, l'évolution de tous les Microchiroptères s'était faite très rapidement ou comme si l'adaptation des Nématodes était postérieure à la diversification des hôtes.

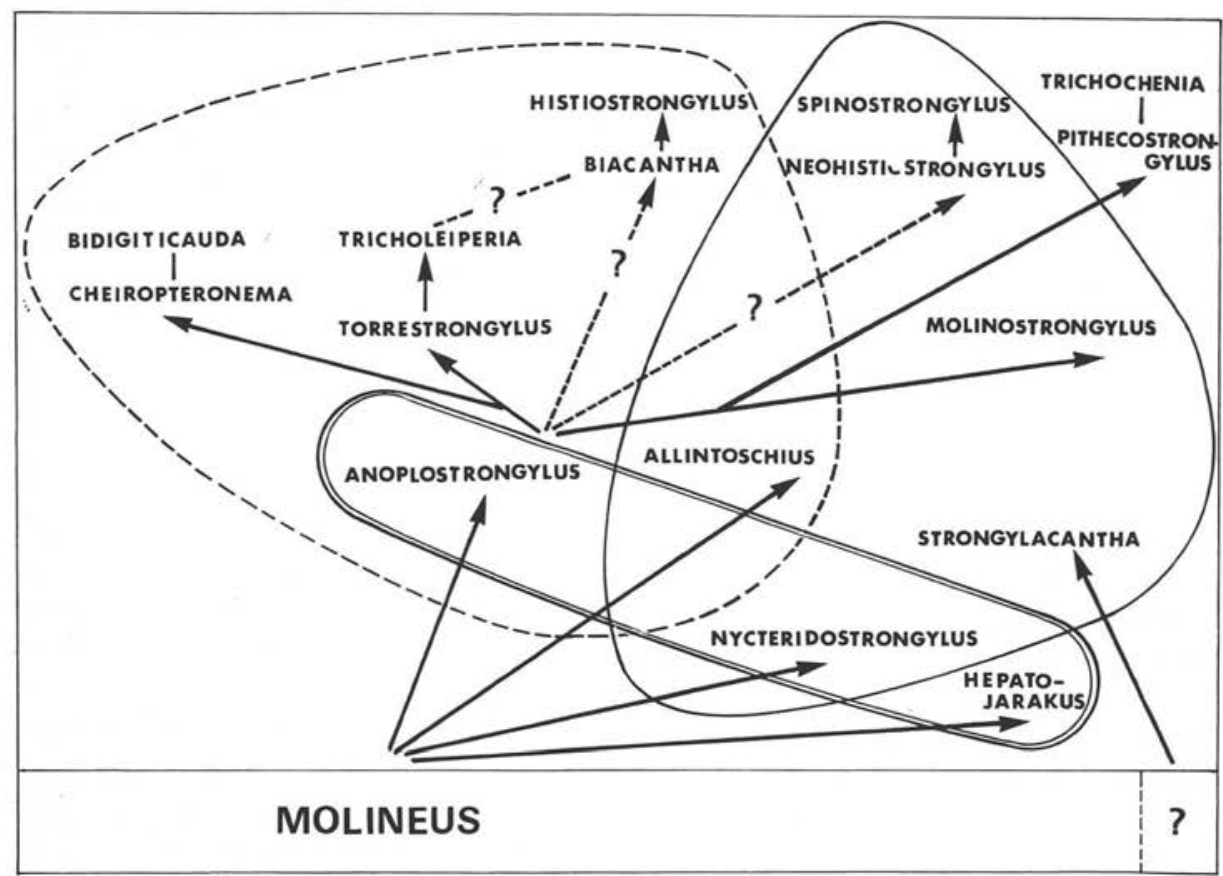

FIG. 12. - Hypothèses sur l'origine et l'évolution des Trichostrongyloidea parasites de Microchiroptères et du Tupaia. Trait simple: Microchiroptères de l'Ancien Monde; trait interrompu: Microchiroptères du Nouveau Monde; double trait: parasites du Tupaia.

\section{VI. - Rapports entre Molineinae et Anoplostrongylinae}

Le seul caractère qui sépare morphologiquement les Molineinae des Anoplostrongylinae réside actuellement dans la différence de l'extrémité caudale des femelles. Les Molineinae ont un filament caudal simple, alors que les Anoplostrongylinae ont, en outre, au moins trois grandes pointes terminales.

La présence de pointes caudales ayant été à juste titre considérée comme un élément primitif, les Anoplostrongylinae ont été généralement considérés comme plus primitifs que les Molineinae. Il apparaît que ce seul élément ne peut suffire à lui seul à déterminer la classification. Des espèces proches les unes des autres et appartenant à un même genre peuvent avoir des pointes caudales chez les Molineinae (Molineus 
vexillarius Dunn, 1961), par exemple et en être dépourvues chez les Anoplostrongylinae [Allintoschius tadaridae (Caballero, 1942), par exemple].

Par ailleurs, l'étude morphologique du synlophe et des bourses caudales montre que les Anoplostrongylinae dérivent des Molineinae et non l'inverse. L'étude du synlophe révèle qu'un genre tel que Pithecostrongylus ayant un simple filament caudal est extrêmement proche de Molinostrongylus.

En conclusion, la famille des Strongylacanthidae ne comporte, en réalité, que le seul genre Strongylacantha, et tous les autres genres (excepté Nicollina, reclassé dans les Amidostomidés par Inglis, 1968), tels qu'ils étaient classés par Chabaud (1960), forment un ensemble complexe avec les Molineinae. Une classification naturelle de cet ensemble devra tenir compte non seulement des pointes caudales des femelles, mais aussi de l'évolution du synlophe et des bourses caudales des mâles.

\section{Bibliographie}

Andreiko (O. F.), Pinchuk (L. M.) et Skvortsov, 1968. - (New species of Nematodes from Microchiroptera). Izv. Akad. Nauk. Moldav. SSR., Ser. Biol. Khim. Nauk., 1, 3-8.

Araujo (T. L. de), 1940. -- Parallintoshius parallintoshius n. g. n. sp. (Nematoda Trichostrongylidae) parasita de Chiroptera. Rev. Fac. Medic. veter., 1, 205-210.

BaER (J. G.), 1959. - Helminthes parasites. Exploration des Parcs Nationaux du Congo Belge. Mission J. G. Baer, W. Gerber (1958), 1, 1-63.

Barus (V.) et RYSAVY (B.). - An analysis of the biogeography of Nematodes of the family Trichostrongylidas parasitizing bats of the suborder Microchiroptera. Folia Parasitologica (Praha), 18, 1-14.

Baylis (H. A.), 1930. - Four new Trichostrongylid Nematodes from Queensland. Ann. Mag. Nat. Hist., ser. 10, 6, 1-18.

Van Bfneden (P. J.), 1873. - Les Parasites des Chauves-souris de Belgique. Mém. Acad. Roy. Sc. Belgique, 40, 1-42.

Brocca (E.) et LE Roux (P.), 1957. - Su un nuovo genere (Angulocirrus gen. nov.) e su due nuove specie di tricostrongilidi (Angulocirrus orycteropi sp. nov., Angulocirrus minor sp. nov.) parassiti di mammiferi africani. Lincei Rend. Sc. fis. mat. e nat., 22, 57-64.

BOULFNGER (C. L.), 1926. - Report on a collection of parasitic Nematodes, mainly from Egypt. Part. IV. Trichostrongylidae and Strongylidae. Parasitology, 18, 86-100.

Brosset (A.), 1966. - La Biologie des Chiroptères. Masson et $C^{1 \mathrm{e}}$, édit., Paris, 240 p.

CABallero (E.), 1942. - Description de Parallintoshius tadaridae n.sp. (Nematoda: Trichostrongylidae) de los murcielagos de Mexico. Ann. Inst. Biol. Mexico, 13, 105-109.

Chabaud (A.-G.), 1960. - Remarques sur la Systématique des Nématodes Trichostrongyloidea. Bull. Soc. Zool. France, 84, 473-483.

Chabaud (A.-G.) et Durette-Desset (M.-C.), 1975. - Remarques sur des Nématodes Trichostrongyloidea qui pourraient indiquer une parenté phylétique entre Tupaiidae et Chiroptères. C.R. Acad. Sci. (Paris), 280, Sér. D, 201-203. 
Chitwood (B. G.), 1937. - A new trichostrongyle Allintoshius nycticeius n. g. n. sp. (Nematoda) from a bat. Proc. Helm. Soc. Washington, 4, 19-20.

CHitwood (B. G.), 1938. - Some Nematodes from the caves of Yucatan. Carnegie Inst. Wash. Publ., 491, 51-66.

Dollfus (R. Ph.), 1954. - Miscellanea helminthologica maroccana XII. Arch. Inst. Pasteur Maroc, 4, 562-582.

DunN (F. L.), 1961. - Molineus vexillarius sp. n. (Nematoda: Trichostrongylidae) from a Peruvian primate, Tamarinus nigricollis (Spix, 1823). J. Parasit., 47, 953-956.

Dunn (F. L.), 1963. - A new Trichostrongylid Nematode from an Oriental Primate. Proc. Helm. Soc. Washington, 30, 161-165.

DURETte-Desser (M.-C.), 1971. - Essai de classification des Nématodes Héligmosomes. Corrélations avec la paléogéographie des hôtes. Mém. Mus. Natn. Hist. Nat., sér. A. Zool., 69, 1-126.

Durette-Desset (M.-C.) et Chabaud (A.-G.), 1975. - Sur trois Nématodes Trichostrongylides parasites de Tupaiidae. Ann. Parasit. hum. comp. (à paraître).

Freitas (J.F. Teixeira de) et Machado de Mendonca (J.), 1960. - Novo nematodeo tricostrongilideo parasito de Quiroptero. Boletin do Museu Paraense Emilio Goeldi, Nov. Sér. Zoologia, 29, 1-7.

INGLIS (W. G.), 1968. - The geographical and evolutionary relationship of Australian trichostrongyloid parasites and their hosts. J. Linn. Soc. (Zool.), 47, 327-347.

KAgei (N.) et SAwada (I.), 1973. - Helminth fauna of bats in Japan XIV. Annotationes Zoologicae Japonenses, 46, 53-56.

Kou (C. C.), 1958. - Studies on parasitic Nematodes of Mammals from Canton. Acta Zoologica Sinica, 10, 79-82.

Lie Kian JoE et Ow YANG, 1963. - Hooperstrongylus orientalis nov. gen., nov. sp. (Nematoda: Trichostrongylidac) from Monkeys. Zeitsch. f. Parasit., 22, 484-488.

Lubimov (M. P.), 1930. - Pithecostrongylus satyri n. g. n. sp. trouvé dans l'intestin d'un orang-outang. Ann. Parasit. hum. comp., 8, 51-56.

MoLIN, 1861. - Il sottordine degli acrofalli ordinato scientificamente secondo i risultamenti delle indagini anatomiche ed embriogeniche. Mem. R. Ist. Veneto Sc. Lett. Arti, 9, 427-633.

MönNig (H. O.), 1927. - Helminthological notes. 11th and 12th Repts. Dir. Vet. Ed. and Res., Pt 1, 224-225.

Morozov (Y.F.) et SPaSsKı (A. A.), 1961. - (Molinostrongylus vespertilionis $\mathrm{n}$. $\mathrm{sp}$. and some morphological characteristics of $M$. alatus and $M$. skrjabini). Helminthologia, 3, 244-250.

ORTLEPP (R. J.), 1932. - Some Helminths from South African Chiroptera. 18th Rep. Direct. Veter. Serv. \& Anim. Ind. Union South Africa, Pretoria, 183-196.

Quentin (J.-C.), 1970. - Description de Dollfustrongylus sciurei n. gen. n. sp., nouveau Trichostrongylide parasite d'un Ecureuil en République Centrafricaine. Cah. La Maboké, 8, 147-151.

SANDGROUND (J. H.), 1929. - Some new parasitic Nematodes from Yucatan (Mexico) including a new genus of Strongyle from cattle. Bull. Mus. Comp. Zool. Harvard, 69, 515-524. 
Schwartz (B.), 1927. - A new parasitic Nematode from an unknown species ot sat. Proc. U.S.N.M., 71, 1-4.

Skarbilovitsch (T.S.), 1934. - Sur la faune des Trichostrongylidae des chauves-souris. Ann. Parasit. hum. comp., 12, 350-361.

Thomas (P. M.), 1959. - Some nematode parasites from Australian hosts. Trans. Roy. Soc. South Australia, 82, 151-162.

Travassos, 1935. - Alguns novos generos e especies de Trichostrongylidae. Rev. Med. Cirurg. Brasil, 43, 345-361.

Vigueras (I. P.), 1935. - Torrestrongylus torrei n. gen. n. sp. parasito de Chiroptera. Mem. Soc. Cubana Hist. Nat. Habana, 9, 57-58.

Wolfgang (R.W.), 1954. - Studies on the endoparasitic fauna of Trinidad mammals. X. Parasites of Chiroptera. Canad. J. Zool., 32, 20-24.

Yamaguti (S.), 1941. - Studies on the Helminth fauna of Japan. Part 35. Mammalian Nematodes II. Jap. J. Zool., 9, 409-438.

Yamashita (J.) et Mori (H.), 1953. - On some species of the endoparasites of bats, Nyctalus maximus aviator Thomas. Mem. Fac. Agric. Hokkaido Univ., 1, 499-503.

YEH (L. S.), 1955. - A new bursate nematode Hepatojarakus malayae gen. et sp. nov. from the liver of Rattus rattus jarak (Bonhote) of Pulan Jarak, Straits of Malacca. J. Helminth., 29, 44-48.

YEH (L. S.), 1957. - Studies on a trematode and a new nematode from a bat from Northern Rhodesia. J. Helminth., 31, 121-125.

Zdzitowiecki (K.), 1970. - Helminths of bats in Poland. IV. Nematodes. Acta Parasit. Pol., $18,255-265$. 